\title{
ADAPTIVE REGULARIZATION, LINEARIZATION, AND DISCRETIZATION AND A POSTERIORI ERROR CONTROL FOR THE TWO-PHASE STEFAN PROBLEM
}

\author{
DANIELE A. DI PIETRO, MARTIN VOHRALÍK, AND SOLEIMAN YOUSEF
}

\begin{abstract}
We consider in this paper the time-dependent two-phase Stefan problem and derive a posteriori error estimates and adaptive strategies for its conforming spatial and backward Euler temporal discretizations. Regularization of the enthalpy-temperature function and iterative linearization of the arising systems of nonlinear algebraic equations are considered. Our estimators yield a guaranteed and fully computable upper bound on the dual norm of the residual, as well as on the $L^{2}\left(L^{2}\right)$ error of the temperature and the $L^{2}\left(H^{-1}\right)$ error of the enthalpy. Moreover, they allow us to distinguish the space, time, regularization, and linearization error components. An adaptive algorithm is proposed, which ensures computational savings through the online choice of a sufficient regularization parameter, a stopping criterion for the linearization iterations, local space mesh refinement, time step adjustment, and equilibration of the spatial and temporal errors. We also prove the efficiency of our estimate. Our analysis is quite general and is not focused on a specific choice of the space discretization and of the linearization. As an example, we apply it to the vertex-centered finite volume (finite element with mass lumping and quadrature) and Newton methods. Numerical results illustrate the effectiveness of our estimates and the performance of the adaptive algorithm.
\end{abstract}

\section{INTRODUCTION}

The two-phase Stefan problem models a phase change process which is governed by the Fourier law; cf. Friedman [22. The two phases, typically solid and liquid, are separated by a moving interface, whose motion is governed by the so-called Stefan condition. Let $\Omega \subset \mathbb{R}^{d}, d \in\{2,3\}$, be an open bounded polygonal or polyhedral domain, not necessarily convex, and let $T>0$. The mathematical statement of the problem is as follows: given an initial enthalpy $u_{0}$ and a source function $f$, find the enthalpy $u$ such that

$$
\begin{aligned}
\partial_{t} u-\nabla \cdot(\nabla \beta(u)) & =f & & \text { in } \Omega \times(0, T), \\
u(\cdot, 0) & =u_{0} & & \text { in } \Omega, \\
\beta(u) & =0 & & \text { on } \partial \Omega \times(0, T) .
\end{aligned}
$$

Received by the editor April 24, 2012 and, in revised form, April 27, 2013.

2010 Mathematics Subject Classification. Primary 65N08, 65N15, 65N50, 80A22.

This work was supported by the ERT project "Enhanced oil recovery and geological sequestration of $\mathrm{CO}_{2}$ : mesh adaptivity, a posteriori error control, and other advanced techniques" (LJLL/IFPEN).

The third author is the corresponding author. 
For the sake of simplicity, we assume that $u$ is normalized so that the (specific) enthalpies of the two phases are 0 and 1 , respectively, and only consider the homogeneous Dirichlet boundary condition (1.1c). The temperature $\beta(u)$ is expressed as a function of the enthalpy $u$. In what follows, we assume that $\beta(\cdot)$ is a nondecreasing Lipschitz continuous function which vanishes in the interval $(0,1)$. The latter condition reflects the latent heat in the phase change.

The numerical analysis of the Stefan problem has been considered in several works. A finite difference method for the multi-dimensional Stefan problem is discussed in Meyer 32. The author presents a convergent numerical scheme which is the implicit analogue of the method of Kamenomostskaja [27. In Ciavaldini [12, the numerical approach is based on finite elements of the first order. The author describes the different schemes used and the nature of their convergence. Elliott [16] presents a finite element approximation of an elliptic variational inequality deduced from a semi-discretization in time of the weak formulation of the two-phase Stefan problem. Discretization schemes for regularized versions of the Stefan problem based on piecewise linear Lagrange finite elements in space and backward differencing in time are presented by Jerome and Rose [25. Jäger and Kačur [24] use the enthalpy formulation and a variational technique to analyze the convergence of linearized semi-discrete-in-time and fully discrete schemes for nonlinear degenerate parabolic systems of porous medium type. In Amiez and Gremaud [2], a numerical scheme based on the approximation of the enthalpy formulation by semi-implicit finite differences in time combined with continuous piecewise linear finite elements in space is presented. Nochetto 33 employs the regularization technique to derive a priori error estimates in $L^{2}\left(0, T ; L^{2}(\Omega)\right)$ for the enthalpy and temperature errors of, respectively, one half and first order for an implicit finite element scheme, under suitable conditions on the data and relation of the space and time steps.

More recently, attention has been paid to finite volume methods which can be used on a large variety of meshes. The framework of semigroup theory has been used by Baughman and Walkington [4] for the study of the co-volume method, which is a special instance of the finite volume method. The analysis predicts one half order rates of convergence for approximate solutions of the enthalpy in $L^{\infty}\left(0, T ; H^{-1}(\Omega)\right)$ and of the temperature in $L^{2}\left(0, T ; L^{2}(\Omega)\right)$. In Eymard et al. [21, the authors give a convergence proof in the case that a finite volume scheme on a general mesh is used for the space discretization. Weak-* convergence for $u$ in $L^{\infty}$ and strong convergence for $\beta(u)$ in $L^{2}$ is shown by means of a priori estimates in $L^{\infty}$ and use of the Kolmogorov theorem on relative compactness of subsets of $L^{2}$. Half order error estimates via regularization have also been obtained in Pop et al. 42, whereas extensions to degenerate hyperbolic-parabolic equations can be found in Andreianov et al. [3].

A technique often used in various numerical approaches (cf. Nochetto [33, Nochetto and Verdi [37, Picasso [40], Beckett et al. 6], or Pop et al. [42]), employs a regularization of the nonsmooth and nonstrictly increasing function $\beta$ by a smooth and strictly increasing one. This allows, in particular, to use the Newton method for the solution of the arising system of nonlinear algebraic equations, albeit its use without regularization has been advocated in Wheeler [52] or in Baughman and Walkington [4] and studied in Kelley and Rulla 28. Alternative approaches such as transformation of dependent variables of Čermák and Zlámal [10] have also been proposed. 
An inevitable tool in practical simulations seems to be an a posteriori error estimate-driven adaptive mesh refinement. One of the first works on a posteriori error estimates for the steady Stefan problem is that of Picasso [40]. Therein, the author derives estimates based on the residual equation for a two-dimensional regularized Stefan problem and proposes a space adaptive finite element algorithm. A posteriori indicators for unsteady phase change problems were derived by Nochetto et al. in 34,35, together with an adaptive algorithm which equilibrates space and time discretization errors. Many other adaptive refinement algorithms such as that of Beckett et al. [6] have also been proposed. Rigorous a posteriori error estimates for nonlinear parabolic problems seem much less developed. In nondegenerate cases, Verfürth [46, 47] was able to obtain an estimator which is both reliable and efficient. A pioneering contribution for degenerate parabolic problems has been obtained by Nochetto et al. in [36]. Therein, $L^{\infty}\left(0, T ; H^{-1}(\Omega)\right)$ estimates for the error in the enthalpy and $L^{2}\left(0, T ; L^{2}(\Omega)\right)$ estimates for the error in the temperature are obtained. The approach is based on the relation of these errors to the residual of (1.1a) obtained through the corresponding dual partial differential equation and subsequent use of the Galerkin orthogonality of the finite element discretization. Recently, rigorous a posteriori error analysis in a space-time dual norm, including some degenerate cases, was given in [14].

The aim of this paper is to derive fully computable a posteriori error estimates and adaptive strategies for the two-phase Stefan problem (1.1) for conforming spatial discretization schemes such as finite element, co-volume, or vertexcentered finite volume schemes with backward Euler time stepping. As in Nochetto et al. [36, our approach is based on the dual norm of the residual. However, we proceed differently in order to have fully and easily computable estimates not featuring any undetermined constants. This is achieved by introducing $\mathbf{H}(\operatorname{div} ; \Omega)$-conforming and locally conservative flux reconstructions following Prager and Synge [43, Ladevèze [29, Destuynder and Métivet [13, Luce and Wohlmuth [30], Braess and Schöberl [8], Repin [45], and [14, 15, 18, 19, 50, see also the references therein.

In Section 2 we give a weak formulation, introduce a regularized problem with a regularization parameter $\epsilon>0$, and fix the notation for temporal and spatial meshes. In Section 3, we identify the residual and its dual norm and we derive an a posteriori error estimate on this problem-dependent error measure. We next split this estimate into estimators characterizing the space, time, regularization, linearization, and quadrature errors.

Section 4 subsequently presents a criterion for the choice of the regularization parameter $\epsilon$ and a stopping criterion for an iterative linearization such as the Newton method. The former is designed to facilitate the treatment of the degeneracy while not spoiling the accuracy, whereas the latter is designed to avoid performing an excessive number of nonlinear solver iterations. These criteria are inspired mainly from [15, 19, 26]. We then propose an adaptive algorithm which uses these criteria while simultaneously performing the usual local mesh refinement and equilibration of the spatial and temporal errors. This algorithm is inspired from [6, 34, 36, 40] and from the work [14, 18, 19,23. We conclude Section 4 by proving that, under these criteria, our estimators are also efficient while representing a lower bound for the dual norm of the residual. 
In Section 5. we show how to bound the $L^{2}\left(0, T ; H^{-1}(\Omega)\right)$-type error in the enthalpy and $L^{2}\left(0, T ; L^{2}(\Omega)\right)$-type error in the temperature by the above dual norm of the residual. In particular, we focus on the use of the Gronwall lemma with as small an overestimation as possible and no appearance of the exponential term $e^{T}$ other than in the approximation of the initial condition. Guaranteed and fully computable a posteriori error estimates on these natural norms immediately follow.

Section 6 presents the application of all these developments to the vertex-centered finite volume (or, equivalently, finite element with mass lumping and numerical quadrature) discretization in space, backward Euler discretization in time, and Newton linearization. Illustrative numerical results fill up Section 7 and, finally, Appendix $\mathrm{A}$ collects the more involved proofs of the various theorems of the paper.

\section{Continuous And Discrete Settings}

This section fixes the basic continuous and discrete settings. More precisely, Section 2.1 presents the continuous problem and the regularization, whereas the basic assumptions on the discretization are introduced in Section 2.2 .

\subsection{Continuous setting.}

2.1.1. The continuous problem. The starting point for our a posteriori analysis is the weak form of problem (1.1). To give it, we need to introduce the assumptions on the data and set up some notation. We suppose that: (i) the enthalpy-temperature function $\beta: \mathbb{R} \rightarrow \mathbb{R}$ is a Lipschitz continuous function such that

$$
\beta(s)=0 \quad \text { in }(0,1),
$$

$\beta$ is strictly increasing in $\mathbb{R}^{-}$and $\mathbb{R}^{+} \backslash(0,1)$, and there exist $c, C>0$ such that, for all $s \in \mathbb{R} \backslash(0,1), \operatorname{sign}(s) \beta(s) \geq c|s|-C$ (see Figure 1); the Lipschitz constant of $\beta$ is denoted by $L_{\beta}$; (ii) the initial enthalpy $u_{0}$ is such that $u_{0} \in L^{2}(\Omega)$; (iii) the source term is such that $f \in L^{2}\left(0, T ; L^{2}(\Omega)\right)$.

We will repeatedly use throughout the paper the two following spaces:

$$
X:=L^{2}\left(0, T ; H_{0}^{1}(\Omega)\right), \quad Z:=H^{1}\left(0, T ; H^{-1}(\Omega)\right) .
$$

We will also need the dual space $X^{\prime}$ of $X$,

$$
X^{\prime}=L^{2}\left(0, T ; H^{-1}(\Omega)\right),
$$

and equip the space $X$ with the norm

$$
\|\varphi\|_{X}:=\left\{\int_{0}^{T}\|\nabla \varphi(\cdot, t)\|_{L^{2}(\Omega)}^{2} \mathrm{~d} t\right\}^{\frac{1}{2}} .
$$

We denote by $\langle\cdot, \cdot\rangle$ the duality pairing between $H^{-1}(\Omega)$ and $H_{0}^{1}(\Omega)$, while $(\cdot, \cdot)_{S}$ is the usual scalar product in $L^{2}(S)$ or $\left[L^{2}(S)\right]^{d}$, with the subscript omitted when $S=\Omega$.

The weak formulation of problem (1.1) can now be stated. It reads: find

$$
u \in Z \quad \text { with } \quad \beta(u) \in X
$$

such that

$$
u(\cdot, 0)=u_{0} \quad \text { in } \Omega
$$

and, for a.e. $s \in(0, T)$,

$$
\left\langle\partial_{t} u(\cdot, s), \varphi\right\rangle+(\nabla \beta(u(\cdot, s)), \nabla \varphi)=(f(\cdot, s), \varphi) \quad \forall \varphi \in H_{0}^{1}(\Omega) .
$$




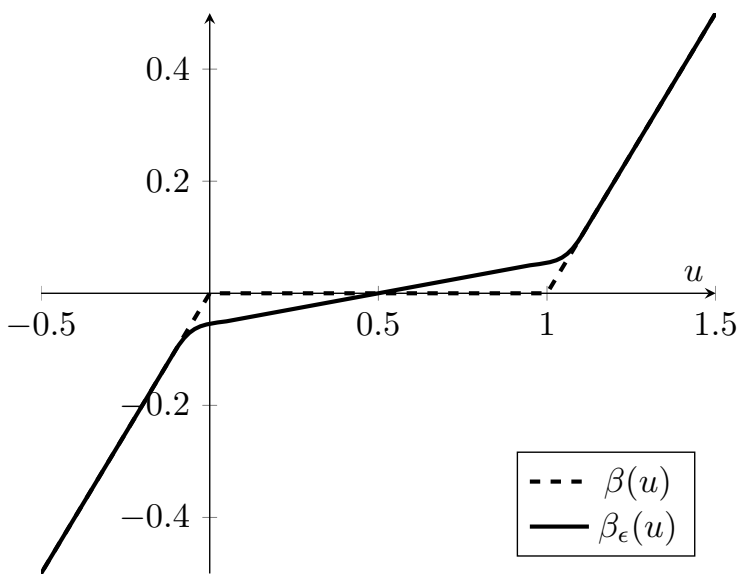

Figure 1. An example of a function $\beta$ and of its regularization $\beta_{\epsilon}$

Existence and uniqueness of the solution to this problem are known [1, $7,22,38$.

2.1.2. A regularization. An important feature of the problem (2.2) is that, as a result of the assumptions on $\beta$, the normal component of the temperature flux $-\nabla \beta(u)$ may jump across the interface

$$
I(t):=\{\mathbf{x} \in \Omega: \beta(u)(\mathbf{x}, t)=0\} .
$$

This fact may hinder both the design and the convergence analysis of a discretization method. Additionally, the lack of smoothness in the dependency of the solution on the problem data can severely affect the convergence of nonlinear iterations. A possible and often employed approach [6, 33, 37, 40, to overcome these difficulties consists in regularizing the problem (2.2) by replacing the function $\beta$ by a smooth, strictly increasing regularized function $\beta_{\epsilon} \in C^{1}(\mathbb{R}), \beta_{\epsilon}^{\prime} \geq \epsilon$, for a parameter $\epsilon>0$; see Figure 1 for an example.

The regularized problem reads as follows: find

$$
u^{\epsilon} \in Z \quad \text { with } \quad \beta_{\epsilon}\left(u^{\epsilon}\right) \in X
$$

such that

$$
u^{\epsilon}(\cdot, 0)=\beta_{\epsilon}^{-1}\left(\beta\left(u_{0}\right)\right) \quad \text { in } \Omega
$$

and, for a.e. $s \in(0, T)$,

$$
\left\langle\partial_{t} u^{\epsilon}(\cdot, s), \varphi\right\rangle+\left(\nabla \beta_{\epsilon}\left(u^{\epsilon}(\cdot, s)\right), \nabla \varphi\right)=(f(\cdot, s), \varphi) \quad \forall \varphi \in H_{0}^{1}(\Omega) .
$$

2.2. Discrete setting. We describe here the basic discrete setting that will be sufficient for the developments of Sections 3 5. Further details are given in Section 6 .

2.2.1. Time mesh. Our focus is on first-order time discretizations based on the backward Euler scheme. Let $\left\{\tau^{n}\right\}_{1 \leq n \leq N}$ denote a sequence of positive real numbers corresponding to the discrete time steps such that $T=\sum_{n=1}^{N} \tau^{n}$. We let $t^{0}:=0$ and, for $1 \leq n \leq N$, we introduce the discrete times $t^{n}:=\sum_{i=1}^{n} \tau^{i}$ and the time intervals $I_{n}:=\left(t^{n-1}, t^{n}\right)$. 
2.2.2. Space meshes. Let $\left\{\mathcal{K}^{n}\right\}_{0 \leq n \leq N}$ denote a family of matching simplicial meshes of the space domain $\Omega$. The initial mesh $\mathcal{K}^{0}$ is used to approximate the initial condition $u_{0}$, while $\mathcal{K}^{n}$ is used to march in time from $t^{n-1}$ to $t^{n}$. The meshes can be refined or coarsened as time evolves. For the developments of Section 4.3 below, we are led to suppose that $\mathcal{K}^{n}, 1 \leq n \leq N$, is obtained from $\mathcal{K}^{n-1}$ by refining some elements and coarsening (a limited number of times) some other ones. We denote by $\mathcal{K}^{n-1, n}$ the coarsest common submesh (overlay) of both $\mathcal{K}^{n}$ and $\mathcal{K}^{n-1}$ and, once again for the developments of Section 4.3. suppose that the meshes $\left\{\mathcal{K}^{n-1, n}\right\}_{1 \leq n \leq N}$ are shape-regular in the sense that there exists a constant $\kappa_{\mathcal{K}}>0$ such that

$$
\min _{K \in \mathcal{K}^{n-1, n}} \frac{\rho_{K}}{h_{K}} \geq \kappa_{\mathcal{K}}
$$

for all $1 \leq n \leq N$, where $\rho_{K}$ denotes the diameter of the largest ball inscribed in the element $K$ and $h_{K}$ the diameter of $K$. For $0 \leq n \leq N$, we denote by $\Pi_{0}^{n}$ the $L^{2}$-orthogonal projection onto the space of piecewise constant functions on $\mathcal{K}^{n}$.

For $0 \leq n \leq N$, let $\mathcal{F}^{n}$ denote the set of mesh faces. Boundary faces are collected in the set $\mathcal{F}^{\mathrm{b}, n}:=\left\{F \in \mathcal{F}^{n} ; F \subset \partial \Omega\right\}$ and we let $\mathcal{F}^{\mathrm{i}, n}:=\mathcal{F}^{n} \backslash \mathcal{F}^{\mathrm{b}, n}$. For a given face $F \in \mathcal{F}^{\mathrm{i}, n}$ we fix an arbitrary orientation and denote the corresponding unit normal vector by $\mathbf{n}_{F}$; for $F \in \mathcal{F}^{\mathrm{b}, n}, \mathbf{n}_{F}$ coincides with the exterior unit normal $\mathbf{n}_{\Omega}$ of $\Omega$. A similar notation for the faces $\mathcal{F}^{n-1, n}$ of the meshes $\mathcal{K}^{n-1, n}$ will also be used.

\section{An a POSTERIORI ERROR ESTIMATE FOR THE DUAL NORM OF THE RESIDUAL}

In this section we derive an a posteriori estimate for the error measured by the dual norm of the residual that we first identify. We then give a basic estimate that we subsequently refine to distinguish the space, time, linearization, regularization, and quadrature errors.

3.1. Dual norm of the residual. As in Picasso [40] or Nochetto et al. 36], our key for deriving a posteriori error estimates for the Stefan problem (1.1) will be the residual and its dual norm. Recall that $u$ denotes the weak solution of the Stefan problem given by (2.2) and the definition of the space $X$ (2.1). Let $u_{h \tau} \in Z$ such that $\beta\left(u_{h \tau}\right) \in X$ is arbitrary. In practice, $u_{h \tau}$ will be the result of the numerical simulation. We define the residual $\mathcal{R}\left(u_{h \tau}\right) \in X^{\prime}$ such that

$$
\begin{aligned}
& \left\langle\mathcal{R}\left(u_{h \tau}\right), \varphi\right\rangle_{X^{\prime}, X} \\
& \quad:=\int_{0}^{T}\left\{\left\langle\partial_{t}\left(u-u_{h \tau}\right), \varphi\right\rangle+\left(\nabla \beta(u)-\nabla \beta\left(u_{h \tau}\right), \nabla \varphi\right)\right\}(s) \mathrm{d} s, \quad \varphi \in X .
\end{aligned}
$$

Using (2.2c), we can infer the following alternative expression for (3.1):

$$
\left\langle\mathcal{R}\left(u_{h \tau}\right), \varphi\right\rangle_{X^{\prime}, X}=\int_{0}^{T}\left\{(f, \varphi)-\left\langle\partial_{t} u_{h \tau}, \varphi\right\rangle-\left(\nabla \beta\left(u_{h \tau}\right), \nabla \varphi\right)\right\}(s) \mathrm{d} s, \quad \varphi \in X .
$$

The norm of the residual in the dual space $X^{\prime}$ is then given by

$$
\left\|\mathcal{R}\left(u_{h \tau}\right)\right\|_{X^{\prime}}:=\sup _{\varphi \in X,\|\varphi\|_{X}=1}\left\langle\mathcal{R}\left(u_{h \tau}\right), \varphi\right\rangle_{X^{\prime}, X} .
$$

The key problem-specific measure of the distance between $u_{h \tau}$ and $u$ that we will use in this paper is given by

$$
\left\|\mathcal{R}\left(u_{h \tau}\right)\right\|_{X^{\prime}}+\left\|u_{0}-u_{h \tau}(\cdot, 0)\right\|_{H^{-1}(\Omega)} .
$$


It follows from (2.2) that the measure (3.3) is zero if and only if the function $u_{h \tau}$ coincides with the exact solution $u$. As we shall see below in Section 5 , it in fact controls the energy error between $u$ and $u_{h \tau}$ and $\beta(u)$ and $\beta\left(u_{h \tau}\right)$. Moreover, this quantity can be easily bounded in terms of error estimators based on $\mathbf{H}(\operatorname{div} ; \Omega)$ conforming flux reconstructions for piecewise affine-in-time $u_{h \tau}$ that we show next.

3.2. General assumptions. In order to proceed with the analysis further, without the necessity to specify at this point any details on how the approximate solution $u_{h \tau}$ was obtained, we are lead to make the following assumption. It requires $Z$ - and $X$-conformity and $u_{h \tau}$ to be piecewise affine and continuous in time on the time $\operatorname{mesh}\left\{I_{n}\right\}_{1 \leq n \leq N}$ of Section 2.2.1

Assumption 3.1 (Approximate solution). The function $u_{h \tau}$ is such that

$$
\begin{aligned}
u_{h \tau} \in Z, \quad \partial_{t} u_{h \tau} \in L^{2}\left(0, T ; L^{2}(\Omega)\right), & \beta\left(u_{h \tau}\right) \in X, \\
\left.u_{h \tau}\right|_{I_{n}} \text { is affine in time on } I_{n} & \forall 1 \leq n \leq N .
\end{aligned}
$$

Note that, consequently, the function $u_{h \tau}$ is uniquely determined by the $N+1$ functions $u_{h}^{n}:=u_{h \tau}\left(\cdot, t^{n}\right), 0 \leq n \leq N$, and $\partial_{t} u_{h \tau}^{n}:=\left.\partial_{t} u_{h \tau}\right|_{I_{n}}=\left(u_{h}^{n}-u_{h}^{n-1}\right) / \tau^{n}$, $1 \leq n \leq N$. We will also employ the abridged notation $u_{h \tau}^{n}$ for $\left.u_{h \tau}\right|_{I_{n}}$.

The second assumption that we make is the existence of a piecewise constantin-time $\mathbf{H}(\operatorname{div} ; \Omega)$-conforming flux reconstruction $\mathbf{t}_{h \tau}$, locally conservative on the meshes $\mathcal{K}^{n}$ of Section 2.2.2. Let us first denote by $\hat{f}$ the piecewise constant-in-time function given by the time-mean values of the source function $f$ on the intervals $I_{n}, 1 \leq n \leq N$.

Assumption 3.2 (Equilibrated flux reconstruction). For all $1 \leq n \leq N$, there exists a vector field $\mathbf{t}_{h}^{n} \in \mathbf{H}(\operatorname{div} ; \Omega)$ such that

$$
\left(\nabla \cdot \mathbf{t}_{h}^{n}, 1\right)_{K}=\left(\hat{f}^{n}, 1\right)_{K}-\left(\partial_{t} u_{h \tau}^{n}, 1\right)_{K} \quad \forall K \in \mathcal{K}^{n} .
$$

We denote by $\mathbf{t}_{h \tau}$ the space-time function such that $\left.\mathbf{t}_{h \tau}\right|_{I_{n}}:=\mathbf{t}_{h}^{n}$ for all $1 \leq n \leq N$.

In Section 6 below, we show how to construct an equilibrated flux reconstruction $\mathbf{t}_{h \tau}$ in the context of vertex-centered finite volume (finite element with mass lumping and quadrature) spatial discretization.

3.3. A basic a posteriori error estimate. We now give an a posteriori error estimate in the general setting of Assumptions 3.1 and 3.2 . Note that the regularization of Section 2.1.2 is not used at the present stage.

We will estimate the error measure (3.3) by the local residual expressed with the flux $\mathbf{t}_{h \tau}$ and by the difference of $\mathbf{t}_{h \tau}$ and the temperature flux, in the spirit of $[8,13,29,30,43,45]$ and $\left[14,15,18,19,50\right.$. More specifically, for $1 \leq n \leq N, \mathbf{t}_{h}^{n}$ as in Assumption 3.2, and $K \in \mathcal{K}^{n}$, we define the residual estimator $\eta_{\mathrm{R}, K}^{n}$ and the flux estimator $\eta_{\mathrm{F}, K}^{n}$ as follows:

$$
\begin{aligned}
\eta_{\mathrm{R}, K}^{n} & :=C_{\mathrm{P}, K} h_{K}\left\|\hat{f}^{n}-\partial_{t} u_{h \tau}^{n}-\nabla \cdot \mathbf{t}_{h}^{n}\right\|_{L^{2}(K)}, \\
\eta_{\mathrm{F}, K}^{n}(t) & :=\left\|\mathbf{t}_{h}^{n}+\nabla \beta\left(u_{h \tau}(\cdot, t)\right)\right\|_{L^{2}(K)} \quad t \in I_{n} .
\end{aligned}
$$

Here, $C_{\mathrm{P}, K}$ is the constant from the Poincaré inequality

$$
\left\|\varphi-\Pi_{0}^{n} \varphi\right\|_{L^{2}(K)} \leq C_{\mathrm{P}, K} h_{K}\|\nabla \varphi\|_{L^{2}(K)} \quad \forall \varphi \in H^{1}(K) .
$$


It holds that $C_{\mathrm{P}, K}=1 / \pi$ as the simplices $K$ are convex; see [5, 39]. Finally, we define the initial condition estimator by

$$
\eta_{\mathrm{IC}}:=\left\|u_{0}-u_{h \tau}(\cdot, 0)\right\|_{H^{-1}(\Omega)} .
$$

We then have:

Theorem 3.3 (A posteriori estimate for the error measure (3.3)). Let $u$ be the weak solution given by (2.2) and let $u_{h \tau}$ and $\mathbf{t}_{h \tau}$ fulfill Assumptions 3.1 and 3.2 , respectively. Then, there holds

$$
\left\|\mathcal{R}\left(u_{h \tau}\right)\right\|_{X^{\prime}}+\left\|u_{0}-u_{h \tau}(\cdot, 0)\right\|_{H^{-1}(\Omega)} \leq \eta+\eta_{\mathrm{IC}},
$$

where

$$
\eta:=\left\{\sum_{n=1}^{N} \int_{I_{n}} \sum_{K \in \mathcal{K}^{n}}\left(\eta_{\mathrm{R}, K}^{n}+\eta_{\mathrm{F}, K}^{n}(t)\right)^{2} \mathrm{~d} t\right\}^{\frac{1}{2}}+\|f-\hat{f}\|_{X^{\prime}}
$$

Proof. Let $\varphi \in X$ with $\|\varphi\|_{X}=1$ be given. Then, adding and subtracting $\left(\mathbf{t}_{h \tau}, \nabla \varphi\right)$ and using Green's theorem, it holds that

$$
\begin{aligned}
\left\langle\mathcal{R}\left(u_{h \tau}\right), \varphi\right\rangle_{X^{\prime}, X} \\
\quad=\int_{0}^{T}\left\{\left(f-\partial_{t} u_{h \tau}-\nabla \cdot \mathbf{t}_{h \tau}, \varphi\right)-\left(\mathbf{t}_{h \tau}+\nabla \beta\left(u_{h \tau}\right), \nabla \varphi\right)\right\}(s) \mathrm{d} s \\
\quad=\int_{0}^{T}\left\{(f-\hat{f}, \varphi)+\left(\hat{f}-\partial_{t} u_{h \tau}-\nabla \cdot \mathbf{t}_{h \tau}, \varphi\right)-\left(\mathbf{t}_{h \tau}+\nabla \beta\left(u_{h \tau}\right), \nabla \varphi\right)\right\}(s) \mathrm{d} s \\
\quad=: \mathfrak{T}_{1}+\mathfrak{T}_{2}+\mathfrak{T}_{3} .
\end{aligned}
$$

For the first term we infer $\mathfrak{T}_{1} \leq\|f-\hat{f}\|_{X^{\prime}}\|\varphi\|_{X}=\|f-\hat{f}\|_{X^{\prime}}$. The second term can be rewritten as follows:

$$
\mathfrak{T}_{2}=\sum_{n=1}^{N} \int_{I_{n}}\left(\hat{f}^{n}-\partial_{t} u_{h \tau}^{n}-\nabla \cdot \mathbf{t}_{h}^{n}, \varphi\right)(s) \mathrm{d} s .
$$

For all $1 \leq n \leq N$ and $t \in I_{n}$, there holds (the dependence of $\varphi$ on the time variable is omitted for brevity),

$$
\begin{aligned}
\left(\hat{f}^{n}-\partial_{t} u_{h \tau}^{n}-\nabla \cdot \mathbf{t}_{h}^{n}, \varphi\right) & =\sum_{K \in \mathcal{K}^{n}}\left(\hat{f}^{n}-\partial_{t} u_{h \tau}^{n}-\nabla \cdot \mathbf{t}_{h}^{n}, \varphi\right)_{K} \\
& =\sum_{K \in \mathcal{K}^{n}}\left(\hat{f}^{n}-\partial_{t} u_{h \tau}^{n}-\nabla \cdot \mathbf{t}_{h}^{n}, \varphi-\Pi_{0}^{n} \varphi\right)_{K} \\
& \leq \sum_{K \in \mathcal{K}^{n}}\left\|\hat{f}^{n}-\partial_{t} u_{h \tau}^{n}-\nabla \cdot \mathbf{t}_{h}^{n}\right\|_{L^{2}(K)}\left\|\varphi-\Pi_{0}^{n} \varphi\right\|_{L^{2}(K)} \\
& \leq \sum_{K \in \mathcal{K}^{n}} C_{\mathrm{P}, K} h_{K}\left\|\hat{f}^{n}-\partial_{t} u_{h \tau}^{n}-\nabla \cdot \mathbf{t}_{h}^{n}\right\|_{L^{2}(K)}\|\nabla \varphi\|_{L^{2}(K)} \\
& =\sum_{K \in \mathcal{K}^{n}} \eta_{\mathrm{R}, K}^{n}\|\nabla \varphi\|_{L^{2}(K)},
\end{aligned}
$$


where we have used the regularity of the arguments, Assumption 3.2 , the CauchySchwarz inequality, and the Poincaré inequality (3.5). For the third term, an application of the Cauchy-Schwarz inequality yields

$$
\mathfrak{T}_{3} \leq \sum_{n=1}^{N} \int_{I_{n}} \sum_{K \in \mathcal{K}^{n}} \eta_{\mathrm{F}, K}^{n}\|\nabla \varphi\|_{L^{2}(K)}(s) \mathrm{d} s .
$$

Collecting the above estimates, using the definition (3.2) of the dual norm of the residual, and using the Cauchy-Schwarz inequality yields (3.7).

3.4. An a posteriori error estimate distinguishing the space, time, regularization, linearization, and quadrature errors. Our next goal is to distinguish the different error components. This is an instrumental step to design an adaptive algorithm where the time step, the space mesh, the regularization parameter, and the stopping criterion for the linearization iterations are chosen optimally. We start by localizing in time the error measure introduced in Section 3.1. For $1 \leq n \leq N$, we let

$$
X_{n}:=L^{2}\left(I_{n} ; H_{0}^{1}(\Omega)\right), \quad Z_{n}:=H^{1}\left(I_{n} ; H^{-1}(\Omega)\right) .
$$

We localize in time the dual norm of the residual (3.2) by setting

$$
\begin{aligned}
& \left\|\mathcal{R}\left(u_{h \tau}\right)\right\|_{X_{n}^{\prime}} \\
& \quad:=\sup _{\varphi \in X_{n},\|\varphi\|_{X_{n}}=1} \int_{I_{n}}\left\{\left\langle\partial_{t}\left(u-u_{h \tau}\right), \varphi\right\rangle+\left(\nabla \beta(u)-\nabla \beta\left(u_{h \tau}\right), \nabla \varphi\right)\right\}(s) \mathrm{d} s .
\end{aligned}
$$

Note that, consequently,

$$
\left\|\mathcal{R}\left(u_{h \tau}\right)\right\|_{X^{\prime}}^{2}=\sum_{n=1}^{N}\left\|\mathcal{R}\left(u_{h \tau}\right)\right\|_{X_{n}^{\prime}}^{2}
$$

for any $u_{h \tau} \in Z$ with $\beta\left(u_{h \tau}\right) \in X$.

Suppose now that we are marching in time from time $t^{n-1}$ to time $t^{n}$ with a given time step $\tau^{n}$, starting from the approximation $u_{h}^{n-1}$. We also suppose that the regularization of Section 2.1.2 has been used for a given value of the parameter $\epsilon$, and that we are on the $k$-th step of some iterative linearization algorithm. We denote by $u_{h}^{n, \epsilon, k}$ the approximation of the solution $u$ at time $t^{n}$ and prescribe the space-time function $u_{h \tau}^{n, \epsilon, k}$ by the value $u_{h}^{n-1}$ at time $t^{n-1}$, by the value $u_{h}^{n, \epsilon, k}$ at time $t^{n}$, and by affine behavior in time on $I_{n}$, i.e.,

$$
u_{h \tau}^{n, \epsilon, k}(\cdot, t)=(1-\rho(t)) u_{h}^{n-1}+\rho(t) u_{h}^{n, \epsilon, k}, \quad \rho(t):=\frac{t-t^{n-1}}{\tau^{n}} .
$$

We summarize our general requirements in the following:

Assumption 3.4 (Adaptive setting). For all $1 \leq n \leq N$, a regularization parameter $\epsilon \geq 0$, and a linearization step $k \geq 1$ :

(i) $u_{h \tau}^{n, \epsilon, k}$ is the approximate solution given by (3.10), $u_{h \tau}^{n, \epsilon, k} \in Z_{n}$ with $\partial_{t} u_{h \tau}^{n, \epsilon, k} \in$ $L^{2}\left(I_{n} ; L^{2}(\Omega)\right)$ and $\beta\left(u_{h \tau}^{n, \epsilon, k}\right) \in X_{n}$

(ii) there exists an equilibrated flux $\mathbf{t}_{h}^{n, \epsilon, k} \in \mathbf{H}(\operatorname{div} ; \Omega)$ such that

$$
\left(\nabla \cdot \mathbf{t}_{h}^{n, \epsilon, k}, 1\right)_{K}=\left(\hat{f}^{n}, 1\right)_{K}-\left(\partial_{t} u_{h \tau}^{n, \epsilon, k}, 1\right)_{K} \quad \forall K \in \mathcal{K}^{n} ;
$$


(iii) $\mathbf{l}_{h}^{n, \epsilon, k} \in\left[L^{2}(\Omega)\right]^{d}$ is the available approximation of the flux $\nabla \beta_{\epsilon}\left(u\left(\cdot, t^{n}\right)\right)$;

(iv) $\Pi^{n}$ is an operator used for interpolatory numerical integration.

An example of the approximate solution $u_{h \tau}^{n, \epsilon, k}$, the linearized flux $\mathbf{l}_{h}^{n, \epsilon, k}$, and the operator $\Pi^{n}$ in the context of the implicit vertex-centered finite volume discretization and Newton linearization is provided in Section 6.3 below.

Proceeding as in Theorem 3.3, it is immediately inferred that

$$
\left\|\mathcal{R}\left(u_{h \tau}^{n, \epsilon, k}\right)\right\|_{X_{n}^{\prime}} \leq\left\{\int_{I_{n}} \sum_{K \in \mathcal{K}^{n}}\left(\eta_{\mathrm{R}, K}^{n, \epsilon, k}+\eta_{\mathrm{F}, K}^{n, \epsilon, k}(t)\right)^{2} \mathrm{~d} t\right\}^{\frac{1}{2}}+\|f-\hat{f}\|_{X_{n}^{\prime}}
$$

where

$$
\begin{aligned}
\eta_{\mathrm{R}, K}^{n, \epsilon, k} & :=C_{\mathrm{P}, K} h_{K}\left\|\hat{f}^{n}-\partial_{t} u_{h \tau}^{n, \epsilon, k}-\nabla \cdot \mathbf{t}_{h}^{n, \epsilon, k}\right\|_{L^{2}(K)}, \\
\eta_{\mathrm{F}, K}^{n, \epsilon, k}(t) & :=\left\|\mathbf{t}_{h}^{n, \epsilon, k}+\nabla \beta\left(u_{h \tau}^{n, \epsilon, k}(\cdot, t)\right)\right\|_{L^{2}(K)},
\end{aligned}
$$

For all $K \in \mathcal{K}^{n}$, we next define the local spatial, temporal, quadrature, regularization, and linearization estimators as follows:

$$
\begin{aligned}
\eta_{\mathrm{sp}, K}^{n, \epsilon, k} & :=\eta_{\mathrm{R}, K}^{n, \epsilon}+\left\|\mathbf{l}_{h}^{n, \epsilon, k}+\mathbf{t}_{h}^{n, \epsilon, k}\right\|_{L^{2}(K)}, \\
\eta_{\mathrm{tm}, K}^{n, \epsilon, k}(t) & :=\left\|\nabla\left(\Pi^{n} \beta\left(u_{h \tau}^{n, \epsilon, k}(\cdot, t)\right)\right)-\nabla\left(\Pi^{n} \beta\left(u_{h}^{n, \epsilon, k}\right)\right)\right\|_{L^{2}(K)}, \quad t \in I_{n}, \\
\eta_{\mathrm{qd}, K}^{n, \epsilon, k}(t) & :=\left\|\nabla\left(\beta\left(u_{h \tau}^{n, \epsilon, k}(\cdot, t)\right)\right)-\nabla\left(\Pi^{n} \beta\left(u_{h \tau}^{n, \epsilon, k}(\cdot, t)\right)\right)\right\|_{L^{2}(K)}, \quad t \in I_{n}, \\
\eta_{\mathrm{reg}, K}^{n, \epsilon, k} & :=\left\|\nabla\left(\Pi^{n} \beta\left(u_{h}^{n, \epsilon, k}\right)\right)-\nabla\left(\Pi^{n} \beta_{\epsilon}\left(u_{h}^{n, \epsilon, k}\right)\right)\right\|_{L^{2}(K)}, \\
\eta_{\mathrm{lin}, K}^{n, \epsilon, k} & :=\left\|\nabla\left(\Pi^{n} \beta_{\epsilon}\left(u_{h}^{n, \epsilon, k}\right)\right)-\mathbf{l}_{h}^{n, \epsilon, k}\right\|_{L^{2}(K)} .
\end{aligned}
$$

Global versions of these estimators are given by

$$
\begin{aligned}
\left(\eta_{\mathrm{sp}}^{n, \epsilon, k}\right)^{2} & :=\tau^{n} \sum_{K \in \mathcal{K}^{n}}\left(\eta_{\mathrm{sp}, K}^{n, \epsilon, k}\right)^{2}, \\
\left(\eta_{\mathrm{tm}}^{n, \epsilon, k}\right)^{2} & :=\int_{I_{n}} \sum_{K \in \mathcal{K}^{n}}\left(\eta_{\mathrm{tm}, K}^{n, \epsilon, k}(t)\right)^{2} \mathrm{~d} t, \\
\left(\eta_{\mathrm{qd}}^{n, \epsilon, k}\right)^{2} & :=\int_{I_{n}} \sum_{K \in \mathcal{K}^{n}}\left(\eta_{\mathrm{qd}, K}^{n, \epsilon, k}(t)\right)^{2} \mathrm{~d} t, \\
\left(\eta_{\mathrm{reg}}^{n, \epsilon, k}\right)^{2} & :=\tau^{n} \sum_{K \in \mathcal{K}^{n}}\left(\eta_{\mathrm{reg}, K}^{n, \epsilon, k}\right)^{2}, \\
\left(\eta_{\mathrm{lin}}^{n, \epsilon, k}\right)^{2} & :=\tau^{n} \sum_{K \in \mathcal{K}^{n}}\left(\eta_{\mathrm{lin}, K}^{n, \epsilon, k}\right)^{2} .
\end{aligned}
$$

Using the inequality (3.12) followed by the triangle inequality we obtain the following estimate:

Corollary 3.5 (Distinguishing the space, time, quadrature, regularization, linearization, and data oscillation errors). Let $u$ be the weak solution given by (2.2), let $1 \leq n \leq N, \epsilon \geq 0$, and $k \geq 1$, and let $u_{h \tau}^{n, \epsilon, k}, \mathbf{t}_{h}^{n, \epsilon, k}, \mathbf{l}_{h}^{n, \epsilon, k}$, and $\Pi^{n}$ be as described in Assumption 3.4. Then there holds

$$
\left\|\mathcal{R}\left(u_{h \tau}^{n, \epsilon, k}\right)\right\|_{X_{n}^{\prime}} \leq \eta_{\mathrm{sp}}^{n, \epsilon, k}+\eta_{\mathrm{tm}}^{n, \epsilon, k}+\eta_{\mathrm{qd}}^{n, \epsilon, k}+\eta_{\mathrm{reg}}^{n, \epsilon, k}+\eta_{\mathrm{lin}}^{n, \epsilon, k}+\|f-\hat{f}\|_{X_{n}^{\prime}} .
$$


Remark 3.6 (Time oscillation of the source term). The error due to the time oscillation of the source term $\|f-\hat{f}\|_{X_{n}^{\prime}}$ is zero provided that the source function $f$ is piecewise constant in time.

\section{BALANCing AND STOPPING CRITERIA, ADAPTive Algorithm, AND EFFICIENCY}

The individual error component estimators of Corollary 3.5 are used in this section to define adaptive criteria to stop the iterative linearizations, to select the value of the regularization parameter $\epsilon$, to locally adapt the quadrature rule, to adjust the time step, and to select the mesh elements to refine/derefine. These criteria are incorporated in a fully adaptive algorithm detailed in Section 4.2. Finally, in Section 4.3 we show the efficiency of our estimators when the adaptive balancing and stopping criteria are used.

4.1. Balancing and stopping criteria. Following [15, 19, 26, this section introduces stopping criteria for the iterative algorithms based on the estimators of Corollary 3.5. The goal is to stop the iterations as soon as the corresponding error component no longer affects significantly the overall error. We assume in what follows that we are marching in time from time $t^{n-1}$ to time $t^{n}$. Let three user-given parameters $\Gamma_{\text {lin }}, \Gamma_{\text {reg }}, \Gamma_{\mathrm{qd}} \in(0,1)$ be given. The criteria are:

(i) Linearization. The linearization iteration is pursued until step $k_{n}$ such that

$$
\eta_{\mathrm{lin}}^{n, \epsilon, k_{n}} \leq \Gamma_{\operatorname{lin}}\left(\eta_{\mathrm{sp}}^{n, \epsilon, k_{n}}+\eta_{\mathrm{tm}}^{n, \epsilon, k_{n}}+\eta_{\mathrm{qd}}^{n, \epsilon, k_{n}}+\eta_{\mathrm{reg}}^{n, \epsilon, k_{n}}\right) .
$$

(ii) Regularization. The regularization parameter $\epsilon$ is reduced until the value $\epsilon_{n}$ such that

$$
\eta_{\mathrm{reg}}^{n, \epsilon_{n}, k_{n}} \leq \Gamma_{\mathrm{reg}}\left(\eta_{\mathrm{sp}}^{n, \epsilon_{n}, k_{n}}+\eta_{\mathrm{tm}}^{n, \epsilon_{n}, k_{n}}+\eta_{\mathrm{qd}}^{n, \epsilon_{n}, k_{n}}\right)
$$

(iii) Quadrature. The quadrature rule is improved until

$$
\eta_{\mathrm{qd}}^{n, \epsilon_{n}, k_{n}} \leq \Gamma_{\mathrm{qd}}\left(\eta_{\mathrm{sp}}^{n, \epsilon_{n}, k_{n}}+\eta_{\mathrm{tm}}^{n, \epsilon_{n}, k_{n}}\right) .
$$

Note that all the linearization, regularization, and quadrature errors may be classified as subsidiary as they can be made as small as desired by increasing the computational effort for fixed mesh and time step; it is thus reasonable to expect that the above criteria will be attained. Local, element by element, versions of the criteria (4.1) -(4.3) can be formulated using the local estimators (3.13) (see [15, 19, 26]), and require that the inequalities hold for all $K \in \mathcal{K}^{n}$; cf. (7.4) for an example.

In the spirit of [36, 41, 48, and [14, 18, 23, we also propose the usual space-time adaptivity:

(iv) Space-time error balancing. The space and time error components should be equilibrated by selecting the time step $\tau^{n}$ and adjusting the spatial meshes $\mathcal{K}^{n}$ in such a way that

$$
\gamma_{\mathrm{tm}} \eta_{\mathrm{sp}}^{n, \epsilon_{n}, k_{n}} \leq \eta_{\mathrm{tm}}^{n, \epsilon_{n}, k_{n}} \leq \Gamma_{\mathrm{tm}} \eta_{\mathrm{sp}}^{n, \epsilon_{n}, k_{n}}
$$

Above, $\Gamma_{\mathrm{tm}}>\gamma_{\mathrm{tm}}>0$ are again user-given parameters, typically close to 1 .

(v) Adaptive mesh refinement. The error in space should be evenly distributed throughout the domain $\Omega$ by local adaptation (refinement, coarsening) of the space mesh $\mathcal{K}^{n}$ in such a way that, for all $K_{1}, K_{2} \in \mathcal{K}^{n}$,

$$
\eta_{\mathrm{sp}, K_{1}}^{n, \epsilon_{n}, k_{n}} \approx \eta_{\mathrm{sp}, K_{2}}^{n, \epsilon_{n}, k_{n}}
$$


In contrast to (4.1)-4.3), the goal is to make $\eta_{\mathrm{sp}}^{n, \epsilon_{n}, k_{n}}$ and $\eta_{\mathrm{tm}}^{n, \epsilon_{n}, k_{n}}$ of comparable size as these error components are substantial and cannot be made arbitrarily small for a given choice of the mesh and of the time step.

4.2. Adaptive algorithm. In this section we propose an adaptive algorithm that implements the balancing and stopping criteria of Section 4.1. Moreover, for a prescribed $\zeta>0$, we aim at satisfying the relation

$$
\frac{\sum_{n=1}^{N}\left\|\mathcal{R}\left(u_{h \tau}\right)\right\|_{X_{n}^{\prime}}^{2}}{\sum_{n=1}^{N}\left\|\mathbf{l}_{h}^{n, \epsilon, k}\right\|_{L^{2}\left(I_{n} ; L^{2}(\Omega)\right)}^{2}} \leq \zeta^{2},
$$

i.e., to bring the relative error under the user-given precision $\zeta$. To account for limited computing resources, we fix refinement thresholds $\underline{h}, \underline{\tau}>0$ for both the mesh size and the time step and require, for all $0 \leq n \leq N$,

$$
\min _{K \in \mathcal{K}^{n}} h_{K} \geq \underline{h}, \quad \tau^{n} \geq \underline{\tau} .
$$

Note that, in particular, because of (4.6), the attainment of (4.5) is not guaranteed.

Recall that $u_{h}^{n, \epsilon, k}$ stands for the approximation of the solution $u_{h}^{n}$ at discrete time $t^{n}$ obtained after $k$ linearization iterations using a regularization parameter $\epsilon$. At each linearization iteration $k$, the new approximation $u_{h}^{n, \epsilon, k}$ is obtained solving the linear problem written schematically as $u_{h}^{n, \epsilon, k}=\Psi\left(u_{h}^{n, \epsilon, k-1}, \tau^{n}, \mathcal{K}^{n}\right)$. For the sake of simplicity, in what follows we neglect the quadrature and data oscillation estimators. Our adaptive algorithm is the following:

Algorithm 4.1 (Adaptive algorithm).

Fix the fractions of cells to refine, $c_{\text {ref }}$, and to derefine, $c_{\text {deref }}$

Choose an initial mesh $\mathcal{K}^{0}$, regularization parameter $\epsilon_{0}$, and a tolerance $\zeta_{\text {IC }}>0$ $u_{h}^{0} \leftarrow \Pi^{0}\left(\beta_{\epsilon_{0}}^{-1}\left(\beta\left(u_{0}\right)\right)\right)$

repeat $\{$ Initial mesh and regularization parameter adaptation $\}$

Compute $\eta_{\mathrm{IC}}$

Refine the cells $K \in \mathcal{K}^{0}$ such that $\eta_{\mathrm{IC}, K} \geq c_{\text {ref }} \max _{L \in \mathcal{K}^{0}}\left\{\eta_{\mathrm{IC}, L}\right\}$ in accordance with (4.6) and adjust the regularization parameter $\epsilon_{0}$

$u_{h}^{0} \leftarrow \Pi^{0}\left(\beta_{\epsilon_{0}}^{-1}\left(\beta\left(u_{0}\right)\right)\right)$

until $\eta_{\mathrm{IC}} \leq \zeta_{\mathrm{IC}}\left\|\nabla\left(\beta_{\epsilon_{0}}\left(u_{h}^{0}\right)\right)\right\|_{L^{2}(\Omega)}$

Choose an initial time step $\tau^{0}$

$\epsilon \leftarrow \epsilon_{0}, t^{0} \leftarrow 0, n \leftarrow 0$

while $t^{n} \leq T$ do $\{$ Time loop $\}$

$n \leftarrow n+1$

$\mathcal{K}^{n} \leftarrow \mathcal{K}^{n-1}$

$\tau^{n} \leftarrow \tau^{n-1}$

$u_{h}^{n, \epsilon, 0} \leftarrow u_{h}^{n-1}$

repeat $\{$ Space refinement $\}$

repeat $\{$ Space and time error balancing $\}$

repeat $\{$ Regularization

$k \leftarrow 0$

repeat Nonlinear solver

$k \leftarrow k+1$

$u_{h}^{n, \epsilon, k}=\Psi\left(u_{h}^{n, \epsilon, k-1}, \tau^{n}, \mathcal{K}^{n}\right)$

Compute $\eta_{\mathrm{sp}}^{n, \epsilon, k}, \eta_{\mathrm{tm}}^{n, \epsilon, k}, \eta_{\mathrm{reg}}^{n, \epsilon, k}, \eta_{\mathrm{lin}}^{n, \epsilon, k}$ 
until (4.1) is satisfied

$k_{n} \leftarrow k$

if (4.2) does not hold then

$\epsilon \leftarrow \epsilon / 2$

end if

until (4.2) is satisfied

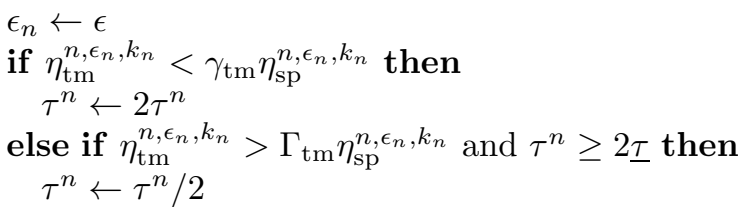

end if

until (4.4) is satisfied or $\tau^{n}=\underline{\tau}$

Refine the cells $K \in \mathcal{K}^{n}$ such that $\eta_{\mathrm{sp}, K}^{n, \epsilon_{n}, k_{n}} \geq c_{\mathrm{ref}} \max _{L \in \mathcal{K}^{n}}\left\{\eta_{\mathrm{sp}, L}^{n, \epsilon_{n}, k_{n}}\right\}$ in accordance with (4.6)

until $\eta_{\mathrm{sp}}^{n, \epsilon_{n}, k_{n}}+\eta_{\mathrm{tm}}^{n, \epsilon_{n}, k_{n}}+\eta_{\mathrm{reg}}^{n, \epsilon_{n}, k_{n}}+\eta_{\mathrm{lin}}^{n, \epsilon_{n}, k_{n}} \leq \zeta\left\|\mathbf{l}_{h}^{n, \epsilon_{n}, k_{n}}\right\|_{L^{2}\left(I_{n} ; L^{2}(\Omega)\right)}$ or $\left(h_{K}=\right.$ $\underline{h}$, for the marked cells)

Derefine the cells $K \in \mathcal{K}^{n}$ such that $\eta_{\mathrm{sp}, K}^{n, \epsilon_{n}, k_{n}} \leq c_{\text {deref }} \max _{L \in \mathcal{K}^{n}}\left\{\eta_{\mathrm{sp}, L}^{n, \epsilon_{n}, k_{n}}\right\}$

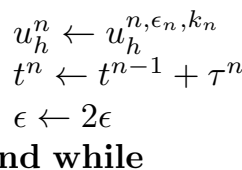

4.3. Efficiency of the a posteriori error estimate. In this section we investigate the global efficiency of the estimators of Corollary [3.5] under the stopping and balancing criteria of Section 4.1. Hence, the quantities at discrete time $t^{n}$ are those obtained after performing $k_{n}$ linearization iterations to meet the criterion (4.1), using a regularization parameter $\epsilon_{n}$ and a quadrature rule such that, respectively, (4.2) and (4.3) are satisfied, and a time step ensuring the time and space error balance (4.4). As usual, in order to use the argument of equivalence of norms on finite-dimensional spaces, we need to assume here:

Assumption 4.2 (Polynomial approximations). For all $1 \leq n \leq N$, the function $u_{h \tau}^{n, \epsilon_{n}, k_{n}}$ is affine in time on the time interval $I_{n}$ and piecewise polynomial of order $m$ in space on the mesh $\mathcal{K}^{n-1, n}$; the functions $\mathbf{l}_{h}^{n, \epsilon_{n}, k_{n}}$ and $\mathbf{t}_{h}^{n, \epsilon_{n}, k_{n}}$ are piecewise polynomial of order $m$ in space on $\mathcal{K}^{n-1, n}$.

For $1 \leq n \leq N$, we introduce the standard residual-based a posteriori error estimators, cf. [48:

$$
\begin{aligned}
\left(\eta_{\mathrm{res}, 1}^{n}\right)^{2} & :=\tau^{n} \sum_{K \in \mathcal{K}^{n-1, n}} h_{K}^{2}\left\|\hat{f}^{n}-\partial_{t} u_{h \tau}^{n, \epsilon_{n}, k_{n}}+\nabla \cdot \mathbf{l}_{h}^{n, \epsilon_{n}, k_{n}}\right\|_{L^{2}(K)}^{2}, \\
\left(\eta_{\mathrm{res}, 2}^{n}\right)^{2} & :=\tau^{n} \sum_{F \in \mathcal{F}^{\mathrm{i}, n-1, n}} h_{F}\left\|\llbracket \mathbf{l}_{h}^{n, \epsilon_{n}, k_{n}} \rrbracket \cdot \mathbf{n}_{F}\right\|_{L^{2}(F)}^{2} .
\end{aligned}
$$

Let $C$ be a generic constant only depending on the shape regularity parameter $\kappa_{\mathcal{K}}$ of the meshes $\mathcal{K}^{n-1, n}, 1 \leq n \leq N$, the space dimension $d$, and the polynomial degree $m$. In order to still proceed generally, without the specification of a particular spatial discretization scheme, we will suppose the following: 
Assumption 4.3 (Approximation property). For all $1 \leq n \leq N$, there holds

$$
\tau^{n} \sum_{K \in \mathcal{K}^{n-1, n}}\left\|\mathbf{1}_{h}^{n, \epsilon_{n}, k_{n}}+\mathbf{t}_{h}^{n, \epsilon_{n}, k_{n}}\right\|_{L^{2}(K)}^{2} \leq C\left(\left(\eta_{\mathrm{res}, 1}^{n}\right)^{2}+\left(\eta_{\mathrm{res}, 2}^{n}\right)^{2}\right) .
$$

This property will be verified in Section [6 below for the vertex-centered finite volume spatial discretization and specific constructions of the fluxes $\mathbf{t}_{h}^{n, \epsilon_{n}, k_{n}}$ and $\mathbf{l}_{h}^{n, \epsilon_{n}, k_{n}}$.

Under these assumptions, we have the following result, showing the equivalence of the error $\left\|\mathcal{R}\left(u_{h \tau}^{n, \epsilon_{n}, k_{n}}\right)\right\|_{X_{n}^{\prime}}$ and the estimators of Corollary 3.5. up to data oscillation:

Theorem 4.4 (Global efficiency). Let, for all $1 \leq n \leq N$, the stopping criteria (4.1) (4.3) as well as the second inequality in the balancing criterion (4.4) be satisfied with the parameters $\Gamma_{\mathrm{lin}}, \Gamma_{\mathrm{reg}}, \Gamma_{\mathrm{qd}}$, and $\Gamma_{\mathrm{tm}}$ small enough. Let Assumptions 4.2 and 4.3 hold true. Then

$\eta_{\mathrm{sp}}^{n, \epsilon_{n}, k_{n}}+\eta_{\mathrm{tm}}^{n, \epsilon_{n}, k_{n}}+\eta_{\mathrm{qd}}^{n, \epsilon_{n}, k_{n}}+\eta_{\mathrm{reg}}^{n, \epsilon_{n}, k_{n}}+\eta_{\mathrm{lin}}^{n, \epsilon_{n}, k_{n}} \leq C\left(\left\|\mathcal{R}\left(u_{h \tau}^{n, \epsilon_{n}, k_{n}}\right)\right\|_{X_{n}^{\prime}}+\|f-\hat{f}\|_{X_{n}^{\prime}}\right)$.

The proof of this result follows the techniques of [49] and the approach of [15]. It is given in Appendix A.1.

\section{AN A POSTERIORI ERROR ESTIMATE FOR THE ERROR IN TEMPERATURE AND ENTHALPY}

In the previous sections we have given a posteriori error estimators for the dual norm of the residual. In this section we prove that these same estimators also bound an error in temperature and enthalpy. We rely on a duality argument which proves to be simpler than using the dual partial differential equation as in [36].

5.1. Bounding the error of the temperature and enthalpy by the dual norm of the residual. For brevity of notation, for $t \in(0, T]$, we let

$$
Q_{t}:=L^{2}\left(0, t ; L^{2}(\Omega)\right), \quad X_{t}:=L^{2}\left(0, t ; H_{0}^{1}(\Omega)\right), \quad X_{t}^{\prime}:=L^{2}\left(0, t ; H^{-1}(\Omega)\right) .
$$

It is convenient to stress that the result of this section applies to all functions $u_{h \tau} \in Z$ such that $\beta\left(u_{h \tau}\right) \in X$. We first state the following bound:

Lemma 5.1 (Simple bounds for the temperature and enthalpy errors). Let $u$ be the solution of (2.2) and let $u_{h \tau} \in Z$ be such that $\beta\left(u_{h \tau}\right) \in X$. Then there holds

$$
\begin{aligned}
\frac{L_{\beta}}{2}\left\|u-u_{h \tau}\right\|_{X^{\prime}}^{2}+\frac{L_{\beta}}{2} \| & \left(u-u_{h \tau}\right)(\cdot, T)\left\|_{H^{-1}(\Omega)}^{2}+\right\| \beta(u)-\beta\left(u_{h \tau}\right) \|_{Q_{T}}^{2} \\
& \leq \frac{L_{\beta}}{2}\left(2 e^{T}-1\right)\left(\left\|\mathcal{R}\left(u_{h \tau}\right)\right\|_{X^{\prime}}^{2}+\left\|u_{0}-u_{h \tau}(\cdot, 0)\right\|_{H^{-1}(\Omega)}^{2}\right)
\end{aligned}
$$

and

$$
\begin{aligned}
& \frac{L_{\beta}}{2}\left\|\left(u-u_{h \tau}\right)(\cdot, T)\right\|_{H^{-1}(\Omega)}^{2}+\left\|\beta(u)-\beta\left(u_{h \tau}\right)\right\|_{Q_{T}}^{2} \\
& \quad \leq \frac{L_{\beta}}{2} e^{T}\left(\left\|\mathcal{R}\left(u_{h \tau}\right)\right\|_{X^{\prime}}^{2}+\left\|u_{0}-u_{h \tau}(\cdot, 0)\right\|_{H^{-1}(\Omega)}^{2}\right) .
\end{aligned}
$$

The results of Lemma 5.1 are classical; we obtain them as a byproduct in the proof of Theorem 5.2 in Section A.2 below. These results are, however, not sufficiently precise. In particular, the use of the Gronwall lemma in its proof implies the appearance of the term $e^{T}$ on the right-hand sides, which grows exponentially with the final time $T$. The purpose of the following theorem is to improve considerably 
this point. Indeed, note that, in Theorem 5.2, the term $e^{T}$ does not appear other than in the approximation of the initial condition $\left\|u_{0}-u_{h \tau}(\cdot, 0)\right\|_{H^{-1}(\Omega)}^{2}$ which can be made sufficiently small. Theorem 5.2 takes a more complicated form than Lemma 5.1 but the numerical results based on its use (see Section 7) prove to be excellent, which is not the case for the framework of Lemma 5.1

Theorem 5.2 (An improved bound for the temperature and enthalpy errors). Let $u$ be the solution of (2.2) and let $u_{h \tau} \in Z$ be such that $\beta\left(u_{h \tau}\right) \in X$. Then there holds

$$
\begin{aligned}
& \frac{L_{\beta}}{2}\left\|u-u_{h \tau}\right\|_{X^{\prime}}^{2}+\frac{L_{\beta}}{2}\left\|\left(u-u_{h \tau}\right)(\cdot, T)\right\|_{H^{-1}(\Omega)}^{2}+\left\|\beta(u)-\beta\left(u_{h \tau}\right)\right\|_{Q_{T}}^{2} \\
& +2 \int_{0}^{T}\left(\left\|\beta(u)-\beta\left(u_{h \tau}\right)\right\|_{Q_{t}}^{2}+\int_{0}^{t}\left\|\beta(u)-\beta\left(u_{h \tau}\right)\right\|_{Q_{s}}^{2} e^{t-s} \mathrm{~d} s\right) \mathrm{d} t \\
\leq & \frac{L_{\beta}}{2}\left\{\left(2 e^{T}-1\right)\left\|u_{0}-u_{h \tau}(\cdot, 0)\right\|_{H^{-1}(\Omega)}^{2}+\left\|\mathcal{R}\left(u_{h \tau}\right)\right\|_{X^{\prime}}^{2}\right. \\
& \left.+2 \int_{0}^{T}\left(\left\|\mathcal{R}\left(u_{h \tau}\right)\right\|_{X_{t}^{\prime}}^{2}+\int_{0}^{t}\left\|\mathcal{R}\left(u_{h \tau}\right)\right\|_{X_{s}^{\prime}}^{2} e^{t-s} \mathrm{~d} s\right) \mathrm{d} t\right\} .
\end{aligned}
$$

The proof of this result is given in Section A.2

5.2. The a posteriori error estimate. The upper bound in Theorem 5.2 can be combined with the results of Section 3.3 to obtain an a posteriori estimate for the temperature and enthalpy errors.

Theorem 5.3 (A posteriori estimate for the temperature and enthalpy errors). Let $u$ be the solution of (2.2) and let $u_{h \tau}$ and $\mathbf{t}_{h \tau}$ fulfill Assumptions 3.1 and 3.2 , respectively. Then there holds

$$
\begin{aligned}
& \frac{L_{\beta}}{2}\left\|u-u_{h \tau}\right\|_{X^{\prime}}^{2}+\frac{L_{\beta}}{2}\left\|\left(u-u_{h \tau}\right)(\cdot, T)\right\|_{H^{-1}(\Omega)}^{2}+\left\|\beta(u)-\beta\left(u_{h \tau}\right)\right\|_{Q_{T}}^{2} \\
& +2 \int_{0}^{T}\left(\left\|\beta(u)-\beta\left(u_{h \tau}\right)\right\|_{Q_{t}}^{2}+\int_{0}^{t}\left\|\beta(u)-\beta\left(u_{h \tau}\right)\right\|_{Q_{s}}^{2} e^{t-s} \mathrm{~d} s\right) \mathrm{d} t \\
\leq & \frac{L_{\beta}}{2}\left\{\left(2 e^{T}-1\right) \eta_{\mathrm{IC}}^{2}+\eta^{2}\right. \\
& \left.+2\left(\sum_{n=1}^{N} \tau^{n} \sum_{l=1}^{n}\left(\eta^{l}\right)^{2}+\sum_{n=1}^{N} \sum_{l=1}^{n} J_{n l}\left\{\sum_{i=1}^{l}\left(\eta^{i}\right)^{2}\right\}\right)\right\},
\end{aligned}
$$

with $\eta_{\mathrm{IC}}$ defined by (3.6), $\eta$ defined by (3.8), $\eta^{n}, 1 \leq n \leq N$, defined by

$$
\eta^{n}:=\left\{\int_{I_{n}} \sum_{K \in \mathcal{K}^{n}}\left(\eta_{\mathrm{R}, K}^{n}+\eta_{\mathrm{F}, K}^{n}(t)\right)^{2} \mathrm{~d} t\right\}^{\frac{1}{2}}+\|f-\hat{f}\|_{X_{n}^{\prime}},
$$

and setting, for $1 \leq n, l \leq N$,

$$
J_{n l}:=\int_{I_{n}} \int_{I_{l}} e^{t-s} \mathrm{~d} s \mathrm{~d} t .
$$


Proof. To prove the result, we rely on Theorem [5.2. Applying Theorem 3.3. it follows that $\left\|\mathcal{R}\left(u_{h \tau}\right)\right\|_{X^{\prime}} \leq \eta$, so we are left to estimate the following right-hand side contributions in terms of the a posteriori error estimators:

$$
\mathfrak{T}_{1}:=\int_{0}^{T}\left\|\mathcal{R}\left(u_{h \tau}\right)\right\|_{X_{t}^{\prime}}^{2} \mathrm{~d} t, \quad \mathfrak{T}_{2}:=\int_{0}^{T}\left(\int_{0}^{t}\left\|\mathcal{R}\left(u_{h \tau}\right)\right\|_{X_{s}^{\prime}}^{2} e^{t-s} \mathrm{~d} s\right) \mathrm{d} t .
$$

As in Theorem 3.3 it is readily inferred that $\left\|\mathcal{R}\left(u_{h \tau}\right)\right\|_{X_{l}^{\prime}} \leq \eta^{l}$ for all $1 \leq l \leq N$, so that

$$
\left\|\mathcal{R}\left(u_{h \tau}\right)\right\|_{X_{t_{n}}^{\prime}}^{2}=\sum_{l=1}^{n}\left\|\mathcal{R}\left(u_{h \tau}\right)\right\|_{X_{l}^{\prime}}^{2} \leq \sum_{l=1}^{n}\left(\eta^{l}\right)^{2} .
$$

Using the fact that $\left\|\mathcal{R}\left(u_{h \tau}\right)\right\|_{X_{t}^{\prime}}$ is a nondecreasing function of the time $t$ together with the above inequality yields for the first term

$$
\mathfrak{T}_{1} \leq \sum_{n=1}^{N} \int_{I_{n}}\left\|\mathcal{R}\left(u_{h \tau}\right)\right\|_{X_{t_{n}}^{\prime}}^{2} \mathrm{~d} t \leq \sum_{n=1}^{N} \int_{I_{n}} \sum_{l=1}^{n}\left(\eta^{l}\right)^{2} \mathrm{~d} t=\sum_{n=1}^{N} \tau^{n} \sum_{l=1}^{n}\left(\eta^{l}\right)^{2} .
$$

Proceeding in a similar way, for the second term $\mathfrak{T}_{2}$ we obtain

$$
\begin{aligned}
\mathfrak{T}_{2} & \leq \sum_{n=1}^{N} \int_{I_{n}} \sum_{l=1}^{n} \int_{I_{l}}\left\|\mathcal{R}\left(u_{h \tau}\right)\right\|_{X_{t_{l}}^{\prime}}^{2} e^{t-s} \mathrm{~d} s \mathrm{~d} t \\
& \leq \sum_{n=1}^{N} \int_{I_{n}} \sum_{l=1}^{n}\left\{\int_{I_{l}} \sum_{i=1}^{l}\left(\eta^{i}\right)^{2} e^{t-s} \mathrm{~d} s\right\} \mathrm{d} t \\
& =\sum_{n=1}^{N} \sum_{l=1}^{n}\left\{\int_{I_{n}} \int_{I_{l}} e^{t-s} \mathrm{~d} s \mathrm{~d} t\right\} \times\left\{\sum_{i=1}^{l}\left(\eta^{i}\right)^{2}\right\}=\sum_{n=1}^{N} \sum_{l=1}^{n} J_{n l}\left\{\sum_{i=1}^{l}\left(\eta^{i}\right)^{2}\right\},
\end{aligned}
$$

whence the conclusion follows.

Remark 5.4 (Simplified versions of the a posteriori estimate). In the spirit of Lemma 5.1, the following simplified versions of the a posteriori estimate of Theorem 5.3 hold:

$$
\begin{aligned}
& \frac{L_{\beta}}{2}\left\|u-u_{h \tau}\right\|_{X^{\prime}}^{2}+\frac{L_{\beta}}{2}\left\|\left(u-u_{h \tau}\right)(\cdot, T)\right\|_{H^{-1}(\Omega)}^{2}+\left\|\beta(u)-\beta\left(u_{h \tau}\right)\right\|_{Q_{T}}^{2} \\
& \quad \leq \frac{L_{\beta}}{2}\left(2 e^{T}-1\right)\left(\eta^{2}+\eta_{\mathrm{IC}}^{2}\right), \\
& \frac{L_{\beta}}{2}\left\|\left(u-u_{h \tau}\right)(\cdot, T)\right\|_{H^{-1}(\Omega)}^{2}+\left\|\beta(u)-\beta\left(u_{h \tau}\right)\right\|_{Q_{T}}^{2} \leq \frac{L_{\beta}}{2} e^{T}\left(\eta^{2}+\eta_{\mathrm{IC}}^{2}\right) .
\end{aligned}
$$

Remark 5.5 (An a posteriori error estimate distinguishing the different error components). While relying on Corollary 3.5 instead of Theorem 3.3. equivalents of Theorem 5.3 and of the bounds of Remark 5.4. distinguishing the different error components can immediately be obtained.

\section{Application to a VerteX-Centered finite volume Discretization}

In this section, we consider the vertex-centered finite volume spatial and backward Euler temporal discretization of the Stefan problem (1.1). The regularization of Section 2.1.2 is considered and the Newton linearization is used. We show how to construct the equilibrated flux $\mathbf{t}_{h}^{n, \epsilon, k}$, the linearized flux $\mathbf{l}_{h}^{n, \epsilon, k}$, and the interpolation operator $\Pi^{n}$ of Assumption 3.4 (in generalization of Assumptions 3.1 and 3.2) and verify Assumptions 4.2 and 4.3 . Thus, all the results of Sections 35 will apply. 

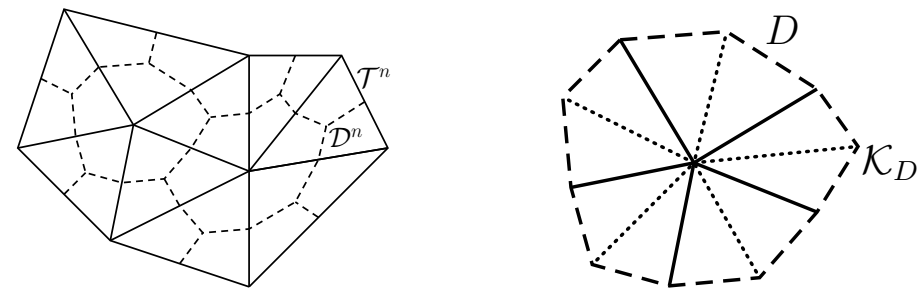

Figure 2. Simplicial mesh $\mathcal{T}^{n}$ and the associated vertex-centered dual mesh $\mathcal{D}^{n}$ (left) and the fine simplicial mesh $\mathcal{K}_{D}$ of $D \in \mathcal{D}^{n}$ (right)

6.1. Dual and tertial space meshes. The vertex-centered finite volume method is defined using a sequence of dual meshes $\left\{\mathcal{D}^{n}\right\}_{0 \leq n \leq N}$ of the space domain $\Omega$. For a given family of matching simplicial primal meshes $\left\{\mathcal{T}^{n}\right\}_{0 \leq n \leq N}$, we construct $\left\{\mathcal{D}^{n}\right\}_{0 \leq n \leq N}$ as follows: for any $0 \leq n \leq N$ and with every vertex a of the mesh $\mathcal{T}^{n}$, we associate one dual volume $D$, constructed by connecting the barycenters of the simplices sharing a through edge (and face for $d=3$ ) barycenters (see Figure2, left) for $d=2$. We split every set $\mathcal{D}^{n}$ into interior dual volumes $\mathcal{D}^{n, \mathrm{i}}$ and boundary dual volumes $\mathcal{D}^{n, \mathrm{~b}}$. The simplicial mesh $\mathcal{K}^{n}$ appearing in Sections 25 is constructed by dividing each $D \in \mathcal{D}^{n}$ into a mesh $\mathcal{K}_{D}$ as indicated in Figure 2, right, if $d=2$ and similarly for $d=3$.

6.2. The vertex-centered finite volume scheme. Let, for $0 \leq n \leq N$,

$$
V_{h}^{n}:=\left\{\varphi_{h} \in C^{0}(\bar{\Omega}) ;\left.\varphi_{h}\right|_{K} \in \mathbb{P}_{1}(K) \quad \forall K \in \mathcal{T}^{n}\right\}
$$

and let

$$
\Pi^{n}: C^{0}(\bar{\Omega}) \rightarrow V_{h}^{n} \quad \text { be the Lagrange interpolation operator }
$$

(cf. Ciarlet [11]), which to a function $\varphi \in C^{0}(\bar{\Omega})$ associates a function $\varphi_{h} \in V_{h}^{n}$ by setting $\varphi_{h}(\mathbf{a}):=\varphi(\mathbf{a})$ for any vertex a of the mesh $\mathcal{T}^{n}$.

Let $u_{h}^{0} \in V_{h}^{0}$ be a suitable approximation of the regularized initial enthalpy $\beta_{\epsilon}^{-1}\left(\beta\left(u_{0}\right)\right)$; see Algorithm 4.1. Next, let $1 \leq n \leq N, u_{h}^{n-1} \in V_{h}^{n-1}$, and a mesh $\mathcal{T}^{n}$ (and consequently $\mathcal{D}^{n}$ ) be given. The vertex-centered finite volume scheme for the regularized Stefan problem (2.3) reads: find $u_{h}^{n, \epsilon} \in V_{h}^{n}$ such that $\beta_{\epsilon}\left(u_{h}^{n, \epsilon}\right)(\mathbf{a})=0$ for all vertices a of $\mathcal{T}^{n}$ on $\partial \Omega$ and such that

$$
\frac{1}{\tau^{n}}\left(u_{h}^{n, \epsilon}-u_{h}^{n-1}, 1\right)_{D}-\left(\nabla \Pi^{n}\left(\beta_{\epsilon}\left(u_{h}^{n, \epsilon}\right)\right) \cdot \mathbf{n}_{D}, 1\right)_{\partial D}=\left(\hat{f}^{n}, 1\right)_{D} \quad \forall D \in \mathcal{D}^{n, \mathrm{i}} .
$$

Then the continuous and piecewise affine-in-time function $u_{h \tau}$ appearing in the previous sections is given by $\left.u_{h \tau}\right|_{I_{n}}:=u_{h \tau}^{n, \epsilon}$,

$$
u_{h \tau}^{n, \epsilon}(\cdot, t)=(1-\rho(t)) u_{h}^{n-1}+\rho(t) u_{h}^{n, \epsilon}, \quad \rho(t):=\frac{t-t^{n-1}}{\tau^{n}} \quad t \in I_{n} .
$$

Remark 6.1 (Regularization). It is also possible to consider the vertex-centered finite volume discretization without any regularization, i.e., use $\beta$ in place of $\beta_{\epsilon}$ in (6.2), with $u_{h}^{0} \in V_{h}^{0}$ an approximation of the initial enthalpy $u_{0}$.

Remark 6.2 (Links to the discretizations of [4,21,36]). Let for simplicity the meshes $\mathcal{T}^{n}$ (and consequently $\mathcal{D}^{n}$ ) do not move in time and let $\hat{f}^{n}$ be piecewise constant on $\mathcal{D}^{n}$. Consider the case without regularization. Then the second and third terms of 
the scheme (6.2) coincide with that of [36, equation (4.4)], because of the links of the vertex-centered finite volumes and finite elements with mass lumping/quadrature for the source term. Similarly, in two space dimensions and when all the angles of $\mathcal{T}^{n}$ are smaller than or equal to $90^{\circ}$, replacing the triangle barycenters by the triangle circumcenters in the construction of $\mathcal{D}^{n}$, the second and third terms of the scheme (6.2) coincide with that in the co-volume method of [4]. More generally, whenever $\mathcal{T}^{n}$ is Delaunay and the mesh $\mathcal{D}^{n}$ is its Voronoï dual, the same link holds true with the cell-centered finite volume scheme of [21]; cf., e.g., [4, 21, 36], [11,20], or [51, Section 3]. Hence the only slight difference between (6.2) and these schemes is in the treatment of the time evolution term which is not mass-lumped herein.

Remark 6.3 (Assumption 3.1). By the definition of $u_{h \tau}$ by (6.2)-(6.3) and by the fact that $u_{h \tau}$ lies in a finite-dimensional space, $u_{h \tau} \in Z$ and $\beta\left(u_{h \tau}\right) \in X$, so that Assumption 3.1 is satisfied. A uniform bound could also be obtained by a priori stability analysis such as those in [4, 21, 36, but is not necessary in our setting.

6.3. Newton linearization. Let $1 \leq n \leq N$ and the mesh $\mathcal{T}^{n}$ (and $\mathcal{D}^{n}$ ) be fixed. Let the vector $G^{n-1}$ be given by its components associated with the dual volumes $D \in \mathcal{D}^{n, \mathrm{i}}, G_{D}^{n-1}:=\left(u_{h}^{n-1}, 1\right)_{D}$, and similarly for the vector $F^{n}, F_{D}^{n}:=\left(\hat{f}^{n}, 1\right)_{D}$. Let $u_{h}^{\mathrm{b}, n, \epsilon} \in V_{h}^{n}$ take the values $\beta_{\epsilon}^{-1}(0)$ (0.5 for the example of Figure1) at the boundary vertices of $\mathcal{T}^{n}$ and the value zero at the other vertices of $\mathcal{T}^{n}$. The last vector that we need is $H^{n, \epsilon}, H_{D}^{n, \epsilon}:=\left(u_{h}^{\mathrm{b}, n, \epsilon}, 1\right)_{D}$. Let, for a given dual volume $E \in \mathcal{D}^{n, \mathrm{i}}$, $\phi_{E}$ stand for the hat basis function of the space $V_{h}^{n}$ associated with $E$; this is a function that takes the value 1 in the vertex associated with $E$ and the value 0 at all other vertices of $\mathcal{T}^{n}$. We also define two matrices, with the components on the line associated with the dual volume $D \in \mathcal{D}^{n, i}$ and on the column associated with the dual volume $E \in \mathcal{D}^{n, \mathrm{i}}$ given by $\mathbb{M}_{D, E}^{n}:=\left(\phi_{E}, 1\right)_{D}, \mathbb{K}_{D, E}^{n}:=\left(\nabla \phi_{E} \cdot \mathbf{n}_{D}, 1\right)_{\partial D}$. All the vectors are of size $\mathbb{R}^{\left|\mathcal{D}^{n, \mathrm{i}}\right|}$ and the matrices of size $\mathbb{R}^{\left|\mathcal{D}^{n, \mathrm{i}}\right| \times\left|\mathcal{D}^{n, \mathrm{i}}\right|}$, with $\left|\mathcal{D}^{n, \mathrm{i}}\right|$ the number of dual volumes in $\mathcal{D}^{n, \mathrm{i}}$ (equal to the number of interior vertices of $\mathcal{T}^{n}$ ). The equation (6.2) can be written in matrix form as follows: find the vector $U^{n, \epsilon}$ such that

$$
\mathbb{M}^{n} U^{n, \epsilon}-\tau^{n} \mathbb{K}^{n} \beta_{\epsilon}\left(U^{n, \epsilon}\right)=\tau^{n} F^{n}+G^{n-1}-H^{n, \epsilon},
$$

where $\left(\beta_{\epsilon}\left(U^{n, \epsilon}\right)\right)_{D}:=\beta_{\epsilon}\left(U_{D}^{n, \epsilon}\right)$. We have $u_{h}^{n, \epsilon}=\sum_{E \in D^{n, \mathrm{i}}} U_{E}^{n, \epsilon} \phi_{E}+u_{h}^{\mathrm{b}, n, \epsilon}$.

The algebraic system (6.4) is nonlinear. Its solution is approximated using the Newton linearization. Let $U^{n, \epsilon, 0}$ be fixed; typically, $U^{n, \epsilon, 0}:=U^{n-1}$. Then, for $k \geq 1$, we approximate

$$
\beta_{\epsilon}\left(U^{n, \epsilon, k}\right) \approx \beta_{\epsilon}\left(U^{n, \epsilon, k-1}\right)+\beta_{\epsilon}^{\prime}\left(U^{n, \epsilon, k-1}\right)\left(U^{n, \epsilon, k}-U^{n, \epsilon, k-1}\right) .
$$

Since the regularized enthalpy-temperature function $\beta_{\epsilon}$ is continuously differentiable, the Newton linearization (6.5) is well defined. At every Newton iteration $k$, we are thus lead to solve the following system of linear algebraic equations: find the vector $U^{n, \epsilon, k}$ such that

$$
\begin{aligned}
\left(\mathbb{M}^{n}\right. & \left.-\tau^{n} \mathbb{K}^{n} \beta_{\epsilon}^{\prime}\left(U^{n, \epsilon, k-1}\right)\right) U^{n, \epsilon, k}=\tau^{n} F^{n}+G^{n-1}-H^{n, \epsilon} \\
& -\tau^{n} \mathbb{K}^{n}\left(\beta_{\epsilon}^{\prime}\left(U^{n, \epsilon, k-1}\right) U^{n, \epsilon, k-1}-\beta_{\epsilon}\left(U^{n, \epsilon, k-1}\right)\right) .
\end{aligned}
$$

At each linearization step $k$, we set

$$
u_{h}^{n, \epsilon, k}:=\sum_{E \in D^{n, \mathrm{i}}} U_{E}^{n, \epsilon, k} \phi_{E}+u_{h}^{\mathrm{b}, n, \epsilon},
$$


which is the function appearing in Section 3.4. The corresponding linearized flux of Assumption 3.4 is given by

$$
\mathbf{l}_{h}^{n, \epsilon, k}:=\nabla\left(\sum_{E \in D^{n, \mathrm{i}}}\left\{\beta_{\epsilon}\left(U_{E}^{n, \epsilon, k-1}\right)+\beta_{\epsilon}^{\prime}\left(U_{E}^{n, \epsilon, k-1}\right)\left(U_{E}^{n, \epsilon, k}-U_{E}^{n, \epsilon, k-1}\right)\right\} \phi_{E}\right) .
$$

We perform the Newton iterations until we meet the convergence criterion discussed in Section 4.1

6.4. Flux reconstruction. Let a time step $1 \leq n \leq N$, a regularization parameter $\epsilon>0$, and a Newton linearization step $k$ be fixed. We now show how to construct the flux $\mathbf{t}_{h}^{n, \epsilon, k}$ of Assumption 3.4. For this purpose, we will solve a local Neumann problem by mixed finite elements on every dual volume, following [17, 30, 51]. For a given $D \in \mathcal{D}^{n}$, we introduce the spaces

$$
\begin{aligned}
\boldsymbol{\operatorname { R T N }}\left(\mathcal{K}_{D}\right) & :=\left\{\mathbf{v}_{h} \in \mathbf{H}(\operatorname{div} ; D) ;\left.\mathbf{v}_{h}\right|_{K} \in\left[\mathbb{P}_{0}(K)\right]^{d}+\mathbf{x} \mathbb{P}_{0}(K) \quad \forall K \in \mathcal{K}_{D}\right\}, \\
\mathbf{R T N}_{\mathrm{N}}\left(\mathcal{K}_{D}\right) & :=\left\{\mathbf{v}_{h} \in \mathbf{R T N}\left(\mathcal{K}_{D}\right) ; \mathbf{v}_{h} \cdot \mathbf{n}_{F}=-\mathbf{l}_{h}^{n, \epsilon, k} \cdot \mathbf{n}_{F} \quad \forall F \in \partial \mathcal{K}_{D}^{\mathrm{i}}\right\}, \\
\mathbf{R T N}_{\mathrm{N}, 0}\left(\mathcal{K}_{D}\right) & :=\left\{\mathbf{v}_{h} \in \mathbf{R T N}\left(\mathcal{K}_{D}\right) ; \mathbf{v}_{h} \cdot \mathbf{n}_{F}=0 \quad \forall F \in \partial \mathcal{K}_{D}^{\mathrm{i}}\right\},
\end{aligned}
$$

where $\partial \mathcal{K}_{D}^{\mathrm{i}}$ stands for all the faces of the submesh $\mathcal{K}_{D}$ which are on the boundary of the dual volume $D$ but not on the boundary of $\Omega$. We will also need the space $\mathbb{P}_{0}^{*}\left(\mathcal{K}_{D}\right)$ which consists of piecewise constants functions on $\mathcal{K}_{D}$; when $D \in \mathcal{D}^{n \text {,i }}$, we additionally impose a zero mean value over $D$. The local problem consists in finding $\mathbf{t}_{h}^{n, \epsilon, k} \in \mathbf{R T N}_{\mathrm{N}}\left(\mathcal{K}_{D}\right)$ and $q_{h} \in \mathbb{P}_{0}^{*}\left(\mathcal{K}_{D}\right)$, the mixed finite element approximations of local Neumann problems on $D \in \mathcal{D}^{n \text {,i }}$ and local Neumann/Dirichlet problems on $D \in \mathcal{D}^{n, \mathrm{~b}}$ :

$$
\begin{aligned}
& \left(\mathbf{t}_{h}^{n, \epsilon, k}+\mathbf{l}_{h}^{n, \epsilon, k}, \mathbf{v}_{h}\right)_{D}-\left(q_{h}, \nabla \cdot \mathbf{v}_{h}\right)_{D}=0 \quad \forall \mathbf{v}_{h} \in \mathbf{R T N}_{\mathrm{N}, 0}\left(\mathcal{K}_{D}\right), \\
& \left(\nabla \cdot \mathbf{t}_{h}^{n, \epsilon, k}, \phi_{h}\right)_{D}-\left(\hat{f}^{n}-\partial_{t} u_{h \tau}^{n, \epsilon, k}, \phi_{h}\right)_{D}=0 \quad \forall \phi_{h} \in \mathbb{P}_{0}^{*}\left(\mathcal{K}_{D}\right) .
\end{aligned}
$$

Note that the problem (6.9) is well-posed and one can take all $\phi_{h} \in \mathbb{P}_{0}\left(\mathcal{K}_{D}\right)$ as the test functions in (6.9b). Indeed, it follows from (6.6) and (6.8) (compare to (6.2)) that

$$
\frac{1}{\tau^{n}}\left(u_{h}^{n, \epsilon, k}-u_{h}^{n-1}, 1\right)_{D}-\left(\mathbf{l}_{h}^{n, \epsilon, k} \cdot \mathbf{n}_{D}, 1\right)_{\partial D}=\left(\hat{f}^{n}, 1\right)_{D} \quad \forall D \in \mathcal{D}^{n, \mathrm{i}} .
$$

From (6.10), we see that the Neumann boundary condition encoded in $\mathbf{R T N}_{\mathrm{N}}\left(\mathcal{K}_{D}\right)$ is in equilibrium with the boundary datum $\hat{f}^{n}-\partial_{t} u_{h \tau}^{n, \epsilon, k}$ of (6.9). We have the following key result:

Lemma 6.4 (Assumptions 3.4, 4.2, and 4.3). Let $1 \leq n \leq N, \epsilon>0$, and $k \geq 1$ be fixed. Let $u_{h}^{n, \epsilon, k}$ be given by (6.6)-(6.7), $\mathbf{l}_{h}^{n, \epsilon, k}$ by (6.8), $\mathbf{t}_{h}^{n, \epsilon, k}$ by (6.9), and $\Pi^{n}$ by (6.1). Then Assumptions 3.4, 4.2, and 4.3 hold true.

Proof. The equilibrium property (3.11) follows immediately from (6.9b), so that Assumption 3.4 is easily satisfied. Whereas Assumption 4.2 is trivial, Assumption 4.3 is obtained by proceeding exactly as in [51, proof of Theorem 5.5] or [15, proof of Lemma 5.3].

\section{NUMERICAL EXPERIMENTS}

We illustrate in this section our theoretical results on a series of numerical experiments for the vertex-centered finite volume discretization approach of Section 6 . 
7.1. Setting. We consider the two-dimensional test case proposed by Nochetto et al. 34, 35] on the space-time domain $\Omega \times(0, T)$ with $\Omega=(0,5)^{2}$ and $T=\pi / 1.25$. The function $\beta(\cdot)$ is given by $\beta(u)=u^{\ominus}+(u-1)^{\oplus}$. The exact temperature has the following expression:

$$
(\beta(u))(x, y, t)= \begin{cases}0.75\left(r^{2}-1\right), & \text { if } r<1, \\ \left(1.5-\rho^{\prime}(t) \frac{y-\rho(t)}{r}\right)(r-1), & \text { if } r \geq 1,\end{cases}
$$

where $r^{2}:=x^{2}+(y-\rho(t))^{2}$ and $\rho(t):=0.5+\sin (1.25 t)$. The exact interface $I(t)$ is a circle with center $(0, \rho(t))$ and radius 1 . The motion of the interface is governed by the Stefan law which prescribes that the normal velocity $\mathbf{v}$ satisfies

$$
\left(\nabla \beta(u)^{+}-\nabla \beta(u)^{-}\right) \cdot \mathbf{n}=\mathbf{v} \quad \text { on } I(t),
$$

where $\nabla \beta(u)^{+}$and $\nabla \beta(u)^{-}$denote the values of the temperature gradient on each side of the interface, while $\mathbf{n}$ is the unit normal to the interface with suitable orientation. The enthalpy $u$ on $\Omega \backslash I(t)$ can be obtained from the expression (7.1) of $\beta(u)$. The homogeneous Neumann condition $\nabla \beta(u) \cdot \mathbf{n}=0$ is enforced at $x=0$, whereas Dirichlet boundary conditions on the temperature are prescribed at $y=0$, $y=5$, and $x=5$ using (7.1). The initial enthalpy $u_{0}$ and the source term $f$ are likewise imposed using (7.1). The vertex-centered finite volume discretization of Section 6 is considered. No adaptation of the quadrature rule is performed; this is a reasonable simplification since the enthalpy-temperature function $\beta(\cdot)$ is piecewise affine.

7.2. Computing approximately the negative norms. In practice we cannot compute the negative norms as the initial data indicator $\eta_{I C}$, the data oscillation $\|f-\hat{f}\|_{X^{\prime}}$, and the dual norm of the residual $\left\|\mathcal{R}\left(u_{h \tau}\right)\right\|_{X^{\prime}}$, even if the exact solution $u$ is known. For numerical experiments below, the dual norms are approximated by solving auxiliary problems. More specifically, for a function $v \in X^{\prime}$ to compute the negative norm $\|v\|_{X^{\prime}}$ we consider for a.e. $t \in(0, T)$ the problem: find $\psi(\cdot, t) \in$ $H_{0}^{1}(\Omega)$ such that

$$
(\nabla \psi(\cdot, t), \nabla \varphi)=\langle v(\cdot, t), \varphi\rangle, \quad \forall \varphi \in H_{0}^{1}(\Omega) .
$$

Then

$$
\begin{aligned}
\|v\|_{X^{\prime}}^{2} & =\int_{0}^{T}\left\{\sup _{\varphi \in H_{0}^{1}(\Omega),\|\nabla \varphi\|_{L^{2}(\Omega)}=1}\langle v(\cdot, t), \varphi\rangle\right\}^{2} \mathrm{~d} t \\
& =\int_{0}^{T}\left\{\sup _{\varphi \in H_{0}^{1}(\Omega),\|\nabla \varphi\|_{L^{2}(\Omega)}=1}(\nabla \psi(\cdot, t), \nabla \varphi)\right\}^{2} \mathrm{~d} t \\
& =\int_{0}^{T}\|\nabla \psi\|_{L^{2}(\Omega)}^{2}(t) \mathrm{d} t=\|\nabla \psi\|_{L^{2}\left(0, T ; L^{2}(\Omega)\right)}^{2} .
\end{aligned}
$$

We obtain an approximation of the function $\psi$ by solving the problem (7.2) numerically by the vertex-centered finite volume scheme on a refined spatial mesh and on discrete times which refine the given temporal mesh. We suppose that the ensuing discretization error is small and can be ignored. The computation of $\eta_{I C}$ is easier as it only involves the initial time $t=0$. 


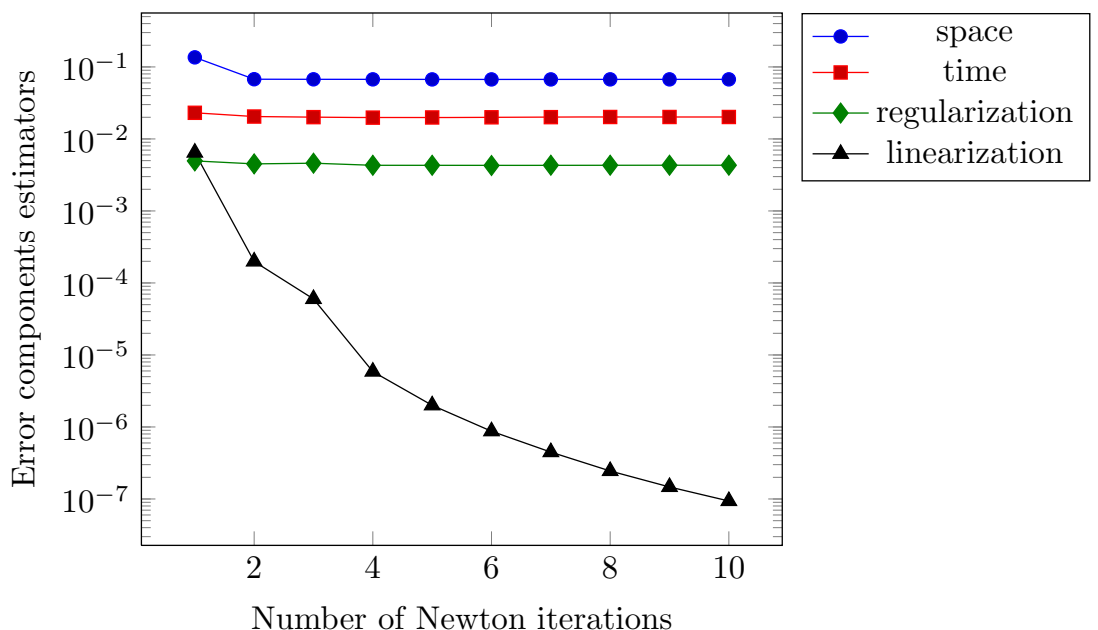

FIgURE 3. Evolution of the spatial, temporal, regularization, and linearization error estimators (3.14) as a function of Newton iterations for a fixed mesh, time step, and regularization parameter

7.3. Stopping criteria. We start by assessing the performance of the balancing and stopping criteria introduced in Section 4.1. Figure 3 depicts the evolution of the spatial (3.14a), temporal (3.14b), regularization (3.14d), and linearization (3.14e) error estimators as a function of the number of Newton iterations for a fixed mesh $\mathcal{K}$ with $h_{\mathcal{K}}=0.25$, time step $\tau=0.1$, and regularization parameter $\epsilon=0.05$. As expected, the linearization error steadily decreases, while the other components stagnate starting from the second iteration. The stopping criterion (4.1) with $\Gamma_{\text {lin }}=$ $10^{-2}$ allows us to profit from this behavior by stopping the Newton algorithm after the second iteration, while a classical criterion based on a fixed threshold,

$$
\eta_{\text {lin }}^{n, \epsilon, k} \leq \zeta_{\text {lin }}, \quad \zeta_{\text {lin }}=10^{-7},
$$

would require 10 iterations to converge. The overall gain for an entire simulation in terms of linearization iterations can be appreciated considering the results in Figure 4, left. We use the adaptive Algorithm 4.1 with different choices for the linearization stopping criterion: the classical criterion (7.3) then the stopping criterion (4.1) with $\Gamma_{\text {lin }}=0.01$ and $\Gamma_{\text {lin }}=0.1$. The other parameters used in the Algorithm are: $\Gamma_{\text {reg }}=0.1, \zeta=1, \zeta_{\mathrm{IC}}=1, h_{\mathcal{K}^{0}}=0.1, \tau^{0}=0.1, \epsilon^{0}=0.25$, $\underline{h}=10^{-2}, \underline{\tau}=10^{-2}, c_{\mathrm{ref}}=0.7, c_{\mathrm{deref}}=0.2, \gamma_{\mathrm{tm}}=0.7$, and $\Gamma_{\mathrm{tm}}=1.3$. For the sake of completeness we also add a comparison with the local version of the stopping criterion (4.1), namely

$$
\eta_{\text {lin }, K}^{n, \epsilon, k_{n}} \leq \Gamma_{\text {lin }, \text { loc }}\left(\eta_{\mathrm{sp}, K}^{n, \epsilon, k_{n}}+\eta_{\mathrm{tm}, K}^{n, \epsilon, k_{n}}+\eta_{\mathrm{qd}, K}^{n, \epsilon, k_{n}}+\eta_{\mathrm{reg}, K}^{n, \epsilon, k_{n}}\right) \quad \forall K \in \mathcal{K}^{n} .
$$

Even with this more stringent criterion, Figure 4 right, shows that a considerable gain in terms of number of linearization iterations can be achieved, whereas the precision on each time step (expressed by our error estimator $\eta^{n}(\underline{5.2})$ ) is basically unchanged. 

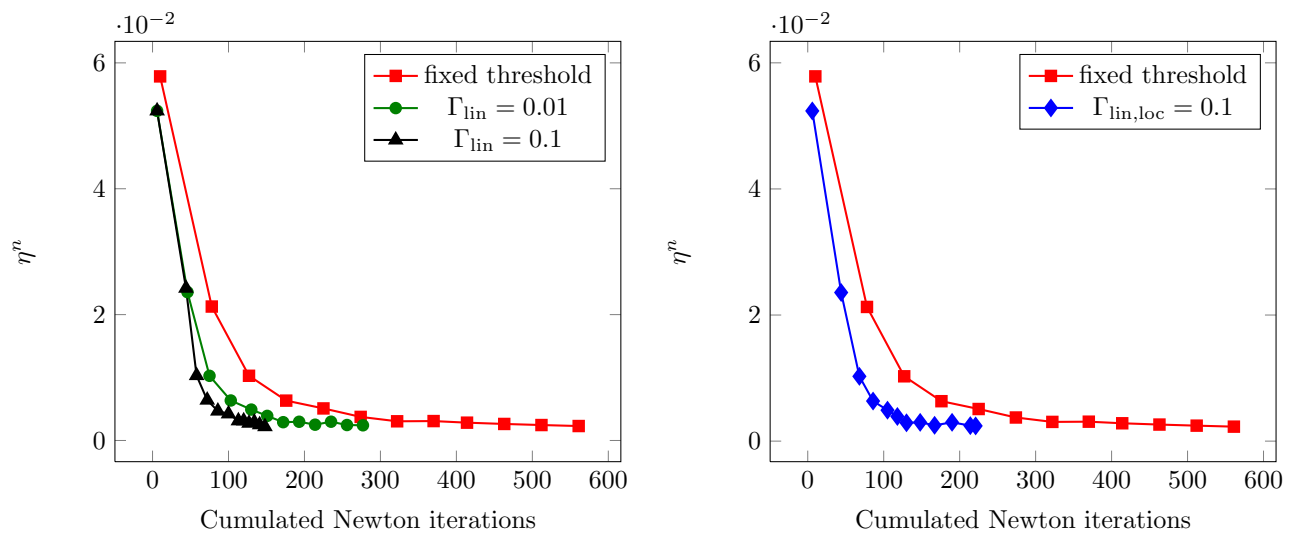

FIGURE 4. Error estimator $\eta^{n}(5.2)$ as a function of the cumulated Newton iterations at each time step (time steps are identified by markers). Global stopping criterion (4.1) or (7.3) (left), local stopping criterion (7.4) (right)

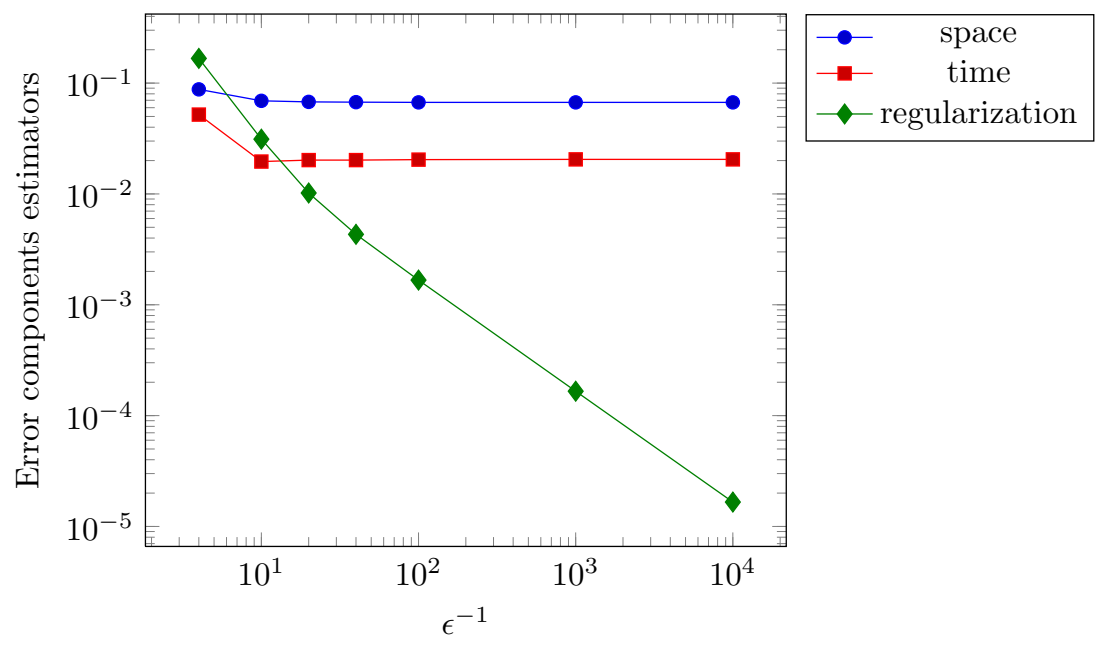

Figure 5. Evolution of the spatial, temporal, and regularization error estimators (3.14) as a function of $\epsilon^{-1}$ for a fixed mesh and time step

Figure 5 shows similar results concerning the criterion (4.2) for the choice of the regularization parameter $\epsilon$ for a fixed mesh $\mathcal{K}$ with $h_{\mathcal{K}}=0.25$ and time step $\tau=0.1$. For each value of $\epsilon$, the Newton iterations are stopped according to (4.1) with $\Gamma_{\text {lin }}=0.1$. The regularization error estimator decreases as expected when $\epsilon$ decreases, while the space and time error estimators stagnate starting from the third iteration. The criterion (4.2) with $\Gamma_{\text {reg }}=0.1$ leads to stopping the iterations after the fourth step. 

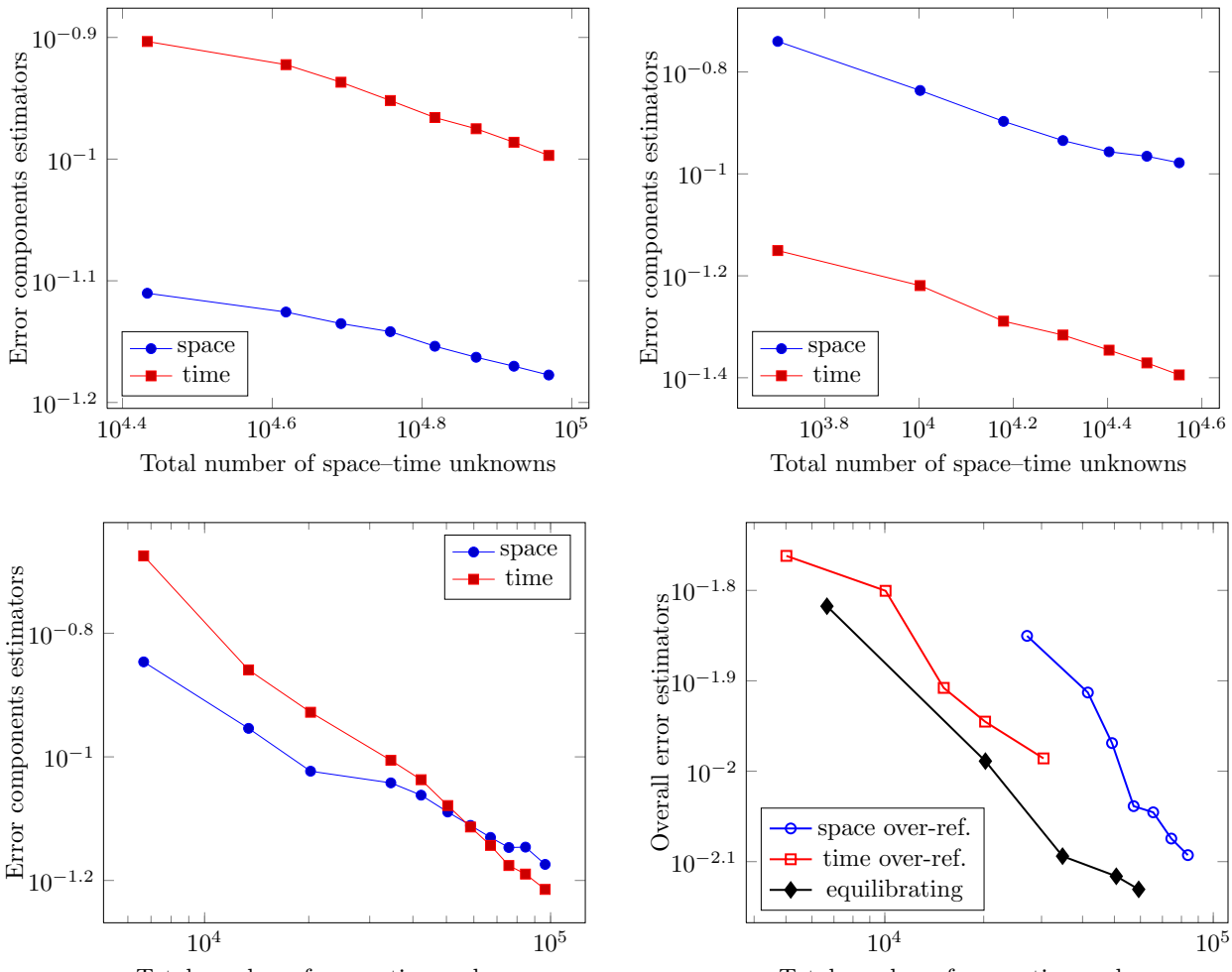

Total number of space-time unknowns

Total number of space-time unknowns

Figure 6. Effect of the time step adaptation strategy on the global error estimator (3.8). Violations of the balancing criterion (4.4) by space over-refinement (top left) and time overrefinement (top right). Time step refinement honoring (4.4) (bottom left). Overall comparison (bottom right)

7.4. Balancing criteria. The next series of numerical experiments aims at assessing the space-time balancing criterion (4.4) by showing its impact on the estimated error (3.8) as a function of the total number of space-time unknowns $\sum_{n=1}^{N}\left|\mathcal{D}^{n, \mathrm{i}}\right|$. In Figure 6, bottom left, we started by an initial mesh $\mathcal{K}^{0}$ with $h_{\mathcal{K}^{0}}=0.4$ and a time step $\tau^{0}=0.1$. Then the time step is adapted in order to satisfy (4.4), with $\gamma_{\mathrm{tm}}=0.7, \Gamma_{\mathrm{tm}}=1.3$. As a result, the spatial (3.14a) and temporal (3.14b) error estimators stay equilibrated during the whole simulation. Figure 6, top, on the other hand, shows two possible disequilibrated patterns corresponding to space and time over-refinement. In the top left we started by an initial mesh $\mathcal{K}^{0}$ with $h_{\mathcal{K}^{0}}=0.2$ and a time step $\tau^{0}=0.2$, we fixed also $\gamma_{\mathrm{tm}}=2$ and $\Gamma_{\mathrm{tm}}=3$, while in the top right we started by an initial mesh $\mathcal{K}^{0}$ with $h_{\mathcal{K}^{0}}=0.5$ and time step $\tau^{0}=0.05$ and we fixed $\gamma_{\mathrm{tm}}=\frac{1}{3}$ and $\Gamma_{\mathrm{tm}}=\frac{1}{2}$. Finally, Figure 6 , bottom right shows the effect of this violating of the balancing criterion (4.4) on the total error. These results make it apparent that the performance of an adaptive code may be considerably reduced when time and space errors are not balanced, and advocate the use of (4.4).

Next, we compare in Figure 7 the actual and predicted error distributions using the adaptive Algorithm 4.1 with $\Gamma_{\text {lin }}=\Gamma_{\text {reg }}=0.1, \zeta=1, \zeta_{\text {IC }}=1, h_{\mathcal{K}^{0}}=0.25$, 

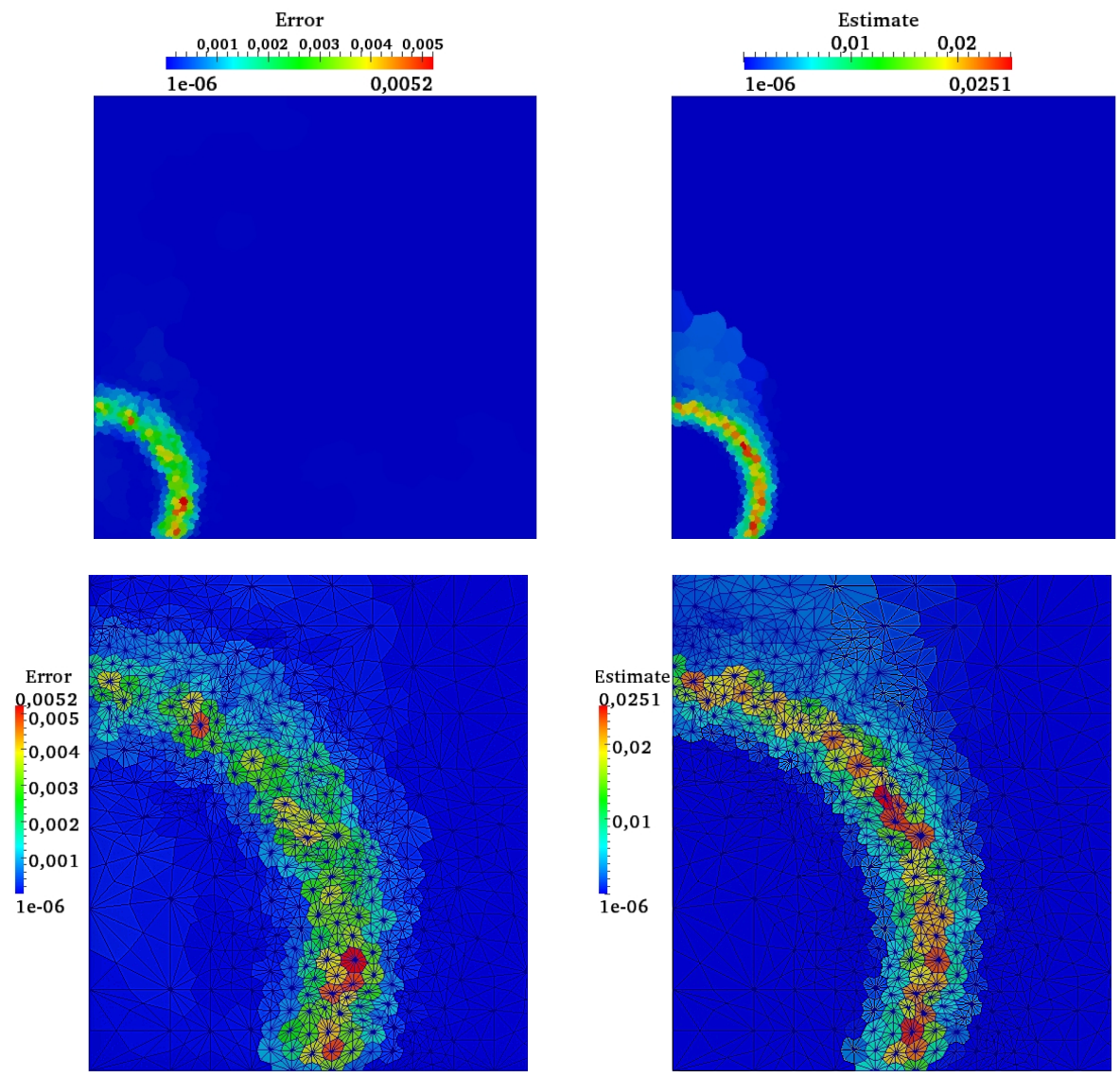

FiguRE 7. Actual (left) and estimated (right) error distribution for $\Gamma_{\text {lin }}=\Gamma_{\text {reg }}=0.1$, adaptive Algorithm 4.1, entire domain (top), interface zoom (bottom)

$\tau^{0}=0.05, \epsilon^{0}=0.25, \underline{h}=10^{-2}, \underline{\tau}=10^{-2}, c_{\mathrm{ref}}=0.7, c_{\mathrm{deref}}=0.2, \gamma_{\mathrm{tm}}=0.7$, and $\Gamma_{\mathrm{tm}}=1.3$. We present the results at time $t=0.1$. We see that the actual and predicted error distributions match very nicely. The corresponding exact and discrete enthalpies are depicted in Figure 8

7.5. Overall performance. In this section we assess the overall performance of the adaptive algorithm of Section 4.2 in terms of precision vs. the number of unknowns.

In Figure 9, left, we depict the error and estimates as a function of the total number of space-time unknowns in the fully adaptive case and in the uniform case. In the adaptive case, we use Algorithm 4.1 with the parameters detailed in Section 7.4. In the uniform case, the temporal and spatial meshes as well as the regularization parameter are fixed during the simulation, and linearization is stopped when (7.3) is satisfied. The error is measured in the dual norm (3.3) and estimated by Theorem 3.3 in the top part of Figure 9, whereas the energylike norm (5.1) and the estimate of Theorem 5.3 are used in the bottom part of 

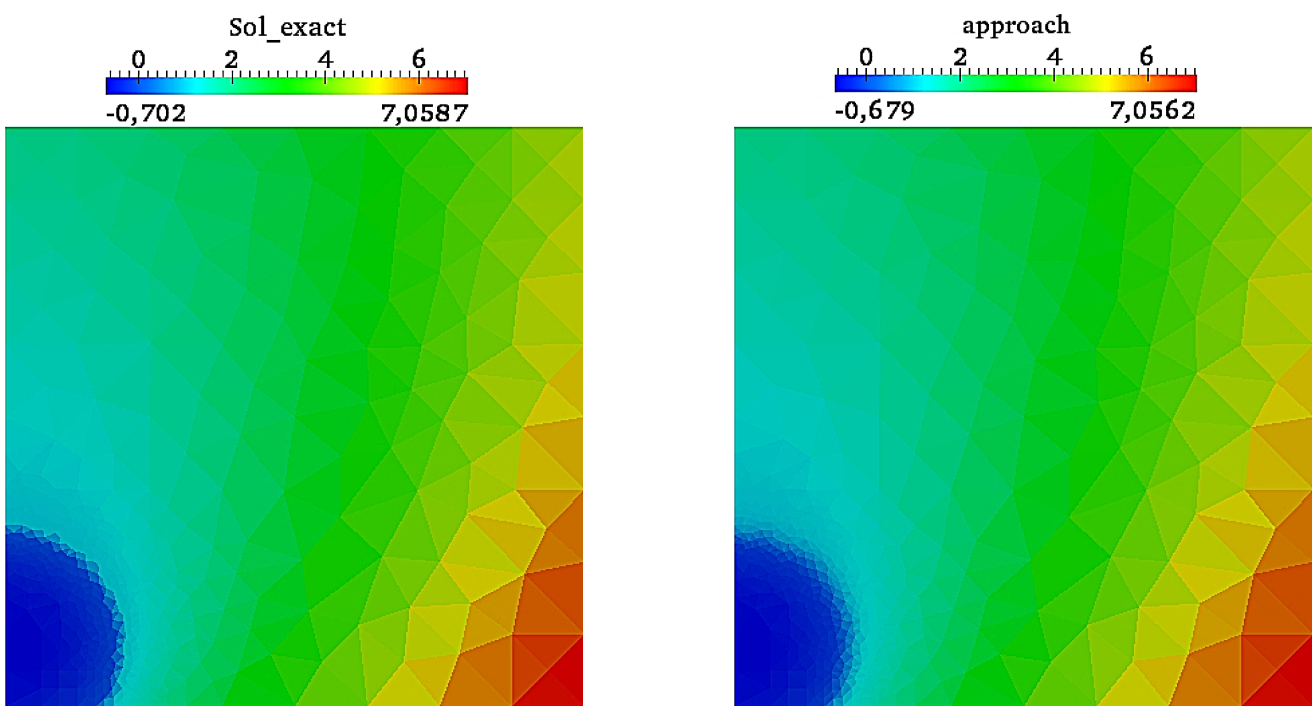

FiguRE 8. Exact (left) and approximate (right) enthalpy corresponding to the results of Figure 7

TABLE 1. Comparison of the experimental orders of convergence (e.o.c.) in the uniform and fully adaptive cases. The total number of space-time unknowns is denoted by $N_{\text {st }}$. The actual error $\left\|\mathcal{R}\left(u_{h \tau}\right)\right\|_{X^{\prime}}$ and the estimated error $\eta$ are defined by (3.2) and (3.8) respectively.

(A) Uniform case

\begin{tabular}{ccccccc}
\hline$N_{\mathrm{st}}$ & $\left\|\beta(u)-\beta\left(u_{h \tau}\right)\right\|_{Q_{T}}$ & e.o.c. & $\left\|\mathcal{R}\left(u_{h \tau}\right)\right\|_{X^{\prime}}$ & e.o.c. & $\eta$ & e.o.c. \\
\hline 7020 & $7.13 \mathrm{e}-02$ & - & $3.75 \mathrm{e}-01$ & - & $1.22 \mathrm{e}-00$ & - \\
66906 & $6.02 \mathrm{e}-02$ & 0.224 & $3.30 \mathrm{e}-01$ & 0.172 & $8.65 \mathrm{e}-01$ & 0.455 \\
915840 & $5.07 \mathrm{e}-02$ & 0.197 & $2.48 \mathrm{e}-01$ & 0.364 & $6.50 \mathrm{e}-01$ & 0.392 \\
$1.12963 \mathrm{e}+07$ & $2.19 \mathrm{e}-02$ & 0.221 & $1.60 \mathrm{e}-01$ & 0.115 & $2.40 \mathrm{e}-01$ & 0.261 \\
\hline
\end{tabular}

(B) Adaptive case

\begin{tabular}{ccccccc}
\hline$N_{\mathrm{st}}$ & $\left\|\beta(u)-\beta\left(u_{h \tau}\right)\right\|_{Q_{T}}$ & e.o.c. & $\left\|\mathcal{R}\left(u_{h \tau}\right)\right\|_{X^{\prime}}$ & e.o.c. & $\eta$ & e.o.c. \\
\hline 9360 & $6.55 \mathrm{e}-02$ & - & $3.51 \mathrm{e}-01$ & - & $1.51 \mathrm{e}-00$ & - \\
35370 & $5.28 \mathrm{e}-02$ & 0.486 & $3.07 \mathrm{e}-01$ & 0.303 & $1.08 \mathrm{e}-00$ & 0.751 \\
224082 & $4.06 \mathrm{e}-02$ & 0.427 & $2.19 \mathrm{e}-01$ & 0.546 & $6.32 \mathrm{e}-01$ & 0.868 \\
$1.53329 \mathrm{e}+06$ & $1.10 \mathrm{e}-02$ & 0.392 & $1.18 \mathrm{e}-01$ & 0.186 & $2.23 \mathrm{e}-01$ & 0.312 \\
\hline
\end{tabular}

Figure 9. In both cases the adaptive strategy yields much better results than the uniform one, as expected. The right part of Figure 9 displays the corresponding effectivity indices, given by the ratio of the estimates over the error. These are remarkably close to the optimal value of one for the dual norm (3.3), even for the 

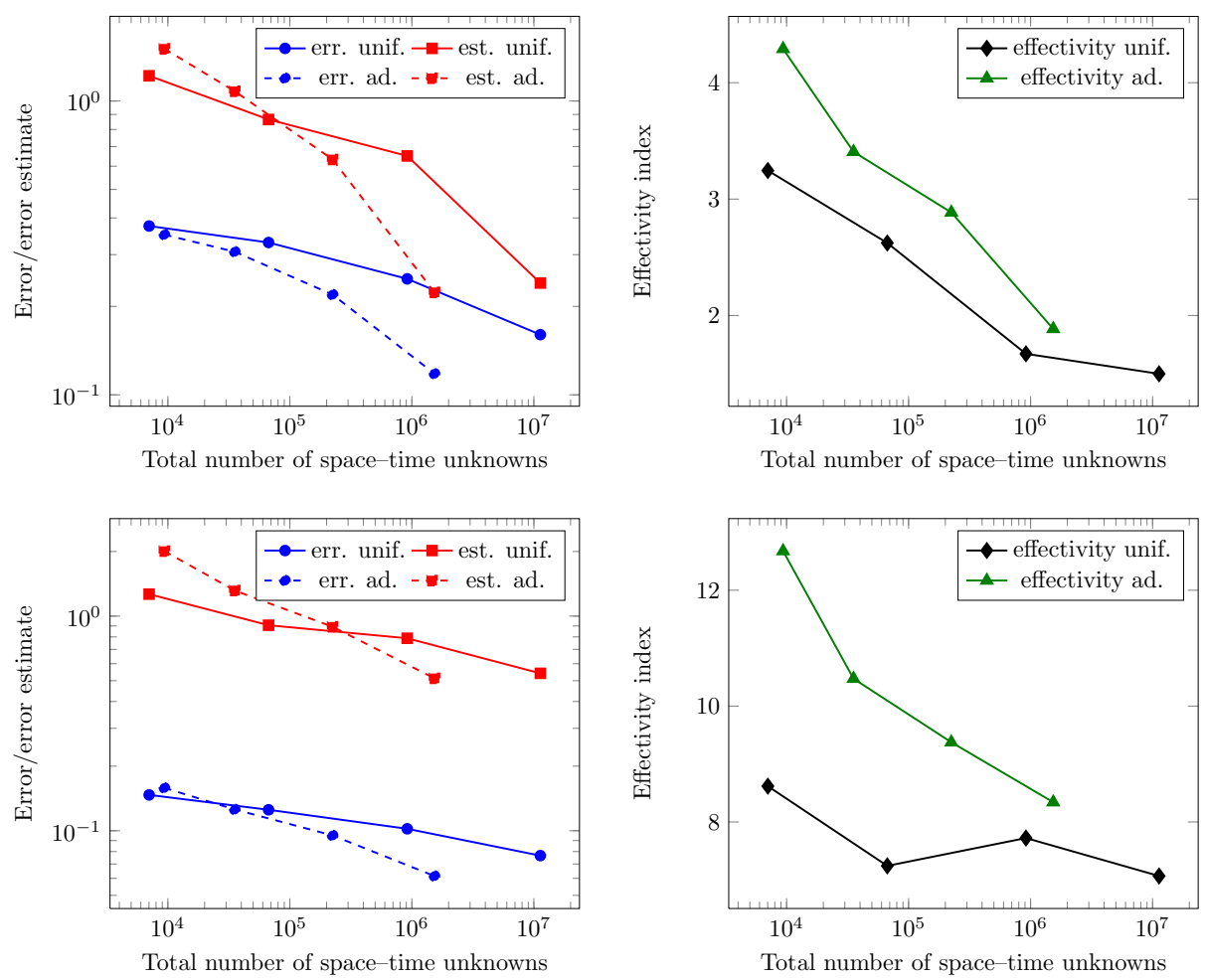

Figure 9. Comparison between adaptive and uniform refinement. Dual norm (3.3) (top), energy-like norm (5.1) (bottom). Error and estimators (left), effectivity indices (right)

present time-dependent, degenerate problem with a moving free boundary. We regard the effectivity indices corresponding to Theorem 5.3 as likewise excellent; there are, in particular, several orders of magnitude smaller than the effectivity indices corresponding to the setting of Remark 5.4 that we have also assessed (not presented).

A quantitative evaluation of the performance in terms of precision vs. the number of unknowns can be obtained by computing the experimental order of convergence (e.o.c.), defined as follows:

$$
\text { e.o.c }:=\frac{\log \left(e_{N_{\mathrm{st}}}\right)-\log \left(e_{M_{\mathrm{st}}}\right)}{-\frac{1}{3}\left(\log N_{\mathrm{st}}-\log M_{\mathrm{st}}\right)},
$$

where $e$ denotes the chosen error measure while $N_{\text {st }}$ and $M_{\text {st }}$ are the total number of space-time unknowns corresponding to two subsequent levels of refinement. The results for the uniform and adaptive cases are collected in Tables $1 \mathrm{a}$ and 1 $\mathrm{b}$, respectively. We evaluate the dual norm of the residual (3.2), the $L^{2}\left(0, T ; L^{2}(\Omega)\right)$ error in the temperature, and the estimator $\eta$ of (3.8). We observe roughly twice faster convergence in the adaptive case in comparison with the uniform one. 


\section{Appendix A. Proofs}

In this appendix, we collect the more involved proofs of some theorems of the paper.

A.1. Proof of Theorem 4.4. In this section, we will use the notation $a \lesssim b$ for the inequality $a \leq C b$ with a generic constant $C$ only depending on the shape regularity parameter $\kappa_{\mathcal{K}}$ of the meshes $\mathcal{K}^{n-1, n}$ and on the maximal level of coarsening between $\mathcal{K}^{n-1}$ and $\mathcal{K}^{n}, 1 \leq n \leq N$, the space dimension $d$, and the polynomial degree $m$. Fix $1 \leq n \leq N$. We start by observing that, owing to the stopping criteria (4.1)-(4.3) and to the second inequality in the balancing criterion (4.4),

$$
\eta_{\mathrm{sp}}^{n, \epsilon_{n}, k_{n}}+\eta_{\mathrm{tm}}^{n, \epsilon_{n}, k_{n}}+\eta_{\mathrm{qd}}^{n, \epsilon_{n}, k_{n}}+\eta_{\mathrm{reg}}^{n, \epsilon_{n}, k_{n}}+\eta_{\mathrm{lin}}^{n, \epsilon_{n}, k_{n}} \lesssim \eta_{\mathrm{sp}}^{n, \epsilon_{n}, k_{n}}
$$

Recall that we have supposed in Section 2.2 .2 that the mesh $\mathcal{K}^{n}, 1 \leq n \leq N$, is obtained from $\mathcal{K}^{n-1}$ by limited refinement/coarsening and that the common refinements $\mathcal{K}^{n-1, n}$ are uniformly shape regular. Thus, for $K \in \mathcal{K}^{n}$, using the triangle inequality, Assumption 4.2, and the inverse inequality, cf. 44, Proposition 6.3 .2 , the first term of (3.13a can be bounded by

$$
\begin{aligned}
& \eta_{\mathrm{R}, K}^{n, \epsilon_{n}, k_{n}}=C_{\mathrm{P}, K} h_{K}\left\|\hat{f}^{n}-\partial_{t} u_{h \tau}^{n, \epsilon_{n}, k_{n}}-\nabla \cdot \mathbf{t}_{h}^{n, \epsilon_{n}, k_{n}}\right\|_{L^{2}(K)} \\
& \leq C_{\mathrm{P}, K} h_{K}\left\|\hat{f}^{n}-\partial_{t} u_{h \tau}^{n, \epsilon_{n}, k_{n}}+\nabla \cdot \mathbf{l}_{h}^{n, \epsilon_{n}, k_{n}}\right\|_{L^{2}(K)} \\
& +C_{\mathrm{P}, K} h_{K}\left\|\nabla \cdot\left(\mathbf{l}_{h}^{n, \epsilon_{n}, k_{n}}+\mathbf{t}_{h}^{n, \epsilon_{n}, k_{n}}\right)\right\|_{L^{2}(K)} \\
& \lesssim\left\{\sum_{K^{\prime} \in \mathcal{K}^{n-1, n}, K^{\prime} \subset K} h_{K^{\prime}}^{2}\left\|\hat{f}^{n}-\partial_{t} u_{h \tau}^{n, \epsilon_{n}, k_{n}}+\nabla \cdot \mathbf{l}_{h}^{n, \epsilon_{n}, k_{n}}\right\|_{L^{2}\left(K^{\prime}\right)}^{2}\right\}^{\frac{1}{2}} \\
& +\left\{\sum_{K^{\prime} \in \mathcal{K}^{n-1, n}, K^{\prime} \subset K}\left\|\mathbf{l}_{h}^{n, \epsilon_{n}, k_{n}}+\mathbf{t}_{h}^{n, \epsilon_{n}, k_{n}}\right\|_{L^{2}\left(K^{\prime}\right)}^{2}\right\}^{\frac{1}{2}} .
\end{aligned}
$$

Consequently, employing Assumption 4.3.

$$
\eta_{\mathrm{sp}}^{n, \epsilon_{n}, k_{n}} \lesssim \eta_{\mathrm{res}, 1}^{n}+\eta_{\mathrm{res}, 2}^{n}
$$

Proving the efficiency of the estimators introduced in Section 3.4 thus amounts to proving the efficiency of the residual estimators $\eta_{\mathrm{res}, 1}^{n}$ and $\eta_{\mathrm{res}, 2}^{n}$.

Henceforth, to simplify, we will use the shorthand notation

$$
u_{h \tau}^{n}=u_{h \tau}^{n, \epsilon_{n}, k_{n}}, \quad \mathbf{l}_{h}^{n}=\mathbf{l}_{h}^{n, \epsilon_{n}, k_{n}}
$$

and denote

$$
\left(\eta_{\mathrm{LRQT}}^{n}\right)^{2}:=\int_{I_{n}} \sum_{K \in \mathcal{K}^{n-1, n}}\left\|\nabla \beta\left(u_{h \tau}^{n}(\cdot, t)\right)-\mathbf{l}_{h}^{n}\right\|_{L^{2}(K)}^{2} \mathrm{~d} t .
$$

We have: 
Lemma A.1 (Estimate of $\left.\eta_{\mathrm{res}, 1}^{n}\right)$. Under the assumptions of Theorem 4.4, there holds

$$
\eta_{\mathrm{res}, 1}^{n} \lesssim\left\|\mathcal{R}\left(u_{h \tau}^{n}\right)\right\|_{X_{n}^{\prime}}+\eta_{\mathrm{LRQT}}^{n}+\|f-\hat{f}\|_{X_{n}^{\prime}}
$$

Proof. For all $K \in \mathcal{K}^{n-1, n}$, we let $v_{K}:=\left.\left(\hat{f}^{n}-\partial_{t} u_{h \tau}^{n}+\nabla \cdot \mathbf{l}_{h}^{n}\right)\right|_{K}$. By Assumption 4.2, $v_{K}$ is polynomial in $K$. We denote by $\psi_{K}$ the usual bubble function on $K$, i.e., the product of the $(d+1)$ hat basis functions (barycentric coordinates) $\psi_{\mathbf{a}}$ associated with the vertices a of the element $K$, set $\lambda_{K}:=h_{K}^{2} \psi_{K} v_{K}$ for all $K \in \mathcal{K}^{n-1, n}$, and let $\lambda:=\sum_{K \in \mathcal{K}^{n-1, n}} \lambda_{K}$. Clearly, $\lambda \in H_{0}^{1}(\Omega)$ and $\left.\lambda\right|_{K} \in H_{0}^{1}(K)$ for all $K \in \mathcal{K}^{n-1, n}$. Using the equivalence of norms on finite-dimensional spaces, integrating by parts in space, the weak form (2.2c), and (3.9) together with the Cauchy-Schwarz inequality; we infer that (cf. [49])

$$
\begin{aligned}
\left(\eta_{\mathrm{res}, 1}^{n}\right)^{2} \lesssim & \int_{I_{n}} \sum_{K \in \mathcal{K}^{n-1, n}} h_{K}^{2}\left(v_{K}, \psi_{K} v_{K}\right)_{K} \mathrm{~d} s \\
= & \int_{I_{n}}\left\{\left\langle\partial_{t}\left(u-u_{h \tau}^{n}\right), \lambda\right\rangle+\left(\nabla \beta(u)-\nabla \beta\left(u_{h \tau}^{n}\right), \nabla \lambda\right)\right. \\
& \left.+\left(\nabla \beta\left(u_{h \tau}^{n}\right)-\mathrm{l}_{h}^{n}, \nabla \lambda\right)+\left(\hat{f}^{n}-f, \lambda\right)\right\} \mathrm{d} s \\
\leq & \left(\left\|\mathcal{R}\left(u_{h \tau}^{n}\right)\right\|_{X_{n}^{\prime}}+\eta_{\mathrm{LRQT}}^{n}+\|f-\hat{f}\|_{X_{n}^{\prime}}\right)\|\lambda\|_{X_{n}} .
\end{aligned}
$$

By the shape regularity of the mesh $\mathcal{K}^{n-1, n}$ and the inverse inequality (cf. 44, Proposition 6.3.2]) we have, for any $K \in \mathcal{K}^{n-1, n}$,

$$
\|\nabla \lambda\|_{L^{2}(K)}=h_{K}^{2}\left\|\nabla\left(\psi_{K} v_{K}\right)\right\|_{L^{2}(K)} \lesssim h_{K}\left\|\psi_{K} v_{K}\right\|_{L^{2}(K)} \leq h_{K}\left\|v_{K}\right\|_{L^{2}(K)} .
$$

An immediate consequence is that $\|\lambda\|_{X_{n}} \lesssim \eta_{\text {res,1 }}^{n}$ and (A.4) follows.

Lemma A.2 (Estimate of $\left.\eta_{\mathrm{res}, 2}^{n}\right)$. Under the assumptions of Theorem 4.4, there holds

$$
\eta_{\mathrm{res}, 2}^{n} \lesssim\left\|\mathcal{R}\left(u_{h \tau}^{n}\right)\right\|_{X_{n}^{\prime}}+\eta_{\mathrm{LRQT}}^{n}+\|f-\hat{f}\|_{X_{n}^{\prime}}
$$

Proof. Let $F \in \mathcal{F}^{\mathrm{i}, n-1, n}$. We denote by $\mathcal{K}_{F}$ the simplices $K \in \mathcal{K}^{n-1, n}$ that share the face $F$. Let $v_{F}:=\llbracket \mathbf{l}_{h}^{n} \rrbracket \cdot \mathbf{n}_{F}$ and keep the same notation for the constant extension of $v_{F}$ into $\mathcal{K}_{F}$ along the vectors face barycenter-opposite vertex. Owing to Assumption 4.2, $v_{F}$ is a polynomial on $\mathcal{K}_{F}$. Let $\psi_{F}$ be the usual face bubble function supported on $\mathcal{K}_{F}$, i.e., the product of the $d$ hat basis functions (barycentric coordinates) $\psi_{\mathbf{a}}$ associated with the vertices a of the face $F$. For all $F \in \mathcal{F}^{\mathrm{i}, n-1, n}$, set $\lambda_{F}:=h_{F} \psi_{F} v_{F}$ and let $\lambda:=\sum_{F \in \mathcal{F}^{\mathrm{i}, n-1, n}} \lambda_{F}$. Note that $\lambda \in H_{0}^{1}(\Omega)$ and $\left.\lambda\right|_{\mathcal{K}_{F}} \in H_{0}^{1}\left(\mathcal{K}_{F}\right)$ for all $F \in \mathcal{F}^{\mathrm{i}, n-1, n}$. Using the equivalence of norms in finitedimensional spaces, integrating by parts in space, using the weak form (2.2C), 
and (3.9) together with the Cauchy-Schwarz inequality, it is inferred that (cf. 49])

$$
\begin{aligned}
\left(\eta_{\mathrm{res}, 2}^{n}\right)^{2} \lesssim & \int_{I_{n}} \sum_{F \in \mathcal{F}^{\mathrm{i}, n-1, n}} h_{F}\left(v_{F}, \psi_{F} v_{F}\right)_{F} \mathrm{~d} s \\
= & \int_{I_{n}} \sum_{F \in \mathcal{F}^{\mathrm{i}, n}-1, n} \sum_{K \in \mathcal{K}_{F}}\left\{\left(\nabla \cdot \mathbf{l}_{h}^{n}, \lambda_{F}\right)_{K}+\left(\mathbf{l}_{h}^{n}, \nabla \lambda_{F}\right)_{K}\right\} \mathrm{d} s \\
= & \int_{I_{n}}\left\{\left(\nabla \cdot \mathbf{l}_{h}^{n}, \lambda\right)+\left(\mathbf{l}_{h}^{n}, \nabla \lambda\right)\right\} \mathrm{d} s \\
= & \int_{I_{n}}\left\{\left\langle\partial_{t}\left(u_{h \tau}^{n}-u\right), \lambda\right\rangle+\left(\nabla \beta\left(u_{h \tau}^{n}\right)-\nabla \beta(u), \nabla \lambda\right)\right. \\
& +\left(\hat{f}^{n}-\partial_{t} u_{h \tau}^{n}+\nabla \cdot \mathbf{l}_{h}^{n}, \lambda\right) \\
& \left.+\left(\mathbf{l}_{h}^{n}-\nabla \beta\left(u_{h \tau}^{n}\right), \nabla \lambda\right)+\left(f-\hat{f}^{n}, \lambda\right)\right\} \mathrm{d} s \\
\lesssim & \left(\left\|\mathcal{R}\left(u_{h \tau}^{n}\right)\right\|_{X_{n}^{\prime}}+\eta_{\mathrm{LRQT}}^{n}+\|f-\hat{f}\|_{X_{n}^{\prime}}\right)\|\lambda\|_{X_{n}} \\
& +\eta_{\mathrm{res}, 1}^{n}\left\{\tau^{n} \sum_{K \in \mathcal{K}^{n-1, n}} h_{K}^{-2}\|\lambda\|_{L^{2}(K)}^{2}\right\}^{\frac{1}{2}} .
\end{aligned}
$$

Using the fact that, for all $F \in \mathcal{F}^{\mathrm{i}, n-1, n}$ and $K \in \mathcal{K}_{F}^{n-1, n},\left\|\psi_{F} v_{F}\right\|_{L^{2}(K)} \lesssim$ $h_{F}^{\frac{1}{2}}\left\|v_{F}\right\|_{L^{2}(F)}$, it is inferred that $\tau^{n} \sum_{K \in \mathcal{K}^{n-1, n},} h_{K}^{-2}\|\lambda\|_{L^{2}(K)}^{2} \lesssim\left(\eta_{\text {res }, 2}^{n}\right)^{2}$, whence by the inverse inequality, $\|\lambda\|_{X_{n}} \lesssim \eta_{\mathrm{res}, 2}^{n}$. Using this fact in (A.7) in conjunction with (A.4), A.6 follows.

Proof of Theorem 4.4. It follows from Lemmas A.1 and A.2 and from (A.2) that

$$
\eta_{\mathrm{sp}}^{n, \epsilon_{n}, k_{n}} \lesssim\left\|\mathcal{R}\left(u_{h \tau}^{n, \epsilon_{n}, k_{n}}\right)\right\|_{X_{n}^{\prime}}+\eta_{\mathrm{LRQT}}^{n}+\|f-\hat{f}\|_{X_{n}^{\prime}} .
$$

In order to bound the term $\eta_{\mathrm{LRQT}}^{n}$, we proceed as follows. The triangle inequality and the definitions (3.13) and (3.14) give

$$
\eta_{\mathrm{LRQT}}^{n} \leq \eta_{\mathrm{lin}}^{n, \epsilon_{n}, k_{n}}+\eta_{\mathrm{reg}}^{n, \epsilon_{n}, k_{n}}+\eta_{\mathrm{qd}}^{n, \epsilon_{n}, k_{n}}+\eta_{\mathrm{tm}}^{n, \epsilon_{n}, k_{n}}
$$

Thus, proceeding as for the bound (A.1),

$$
\eta_{\mathrm{LRQT}}^{n} \leq C \eta_{\mathrm{sp}}^{n, \epsilon_{n}, k_{n}},
$$

where the constant $C$ only depends on the parameters $\Gamma_{\text {lin }}, \Gamma_{\text {reg }}$, and $\Gamma_{\mathrm{qd}}$ in the stopping criteria (4.1)- (4.3) and $\Gamma_{\mathrm{tm}}$ in the balancing criterion (4.4). Thus, choosing these parameters small enough, the term $\eta_{\mathrm{LRQT}}^{n}$ can be made small enough to be discarded from the right-hand side of (A.8); cf. [15. Theorem 4.4] and the assertion of Theorem 4.4 follows from (A.1).

A.2. Proof of Theorem [5.2, We start by proving the following intermediate result.

Lemma A.3 (Duality bound). Let $u$ be the solution to (2.2) and let $u_{h \tau} \in Z$ be such that $\beta\left(u_{h \tau}\right) \in X$. Then, there holds, for a.e. $t \in(0, T)$,

$$
\begin{aligned}
& \frac{2}{L_{\beta}}\left\|\beta(u)-\beta\left(u_{h \tau}\right)\right\|_{Q_{t}}^{2}+\left\|\left(u-u_{h \tau}\right)(\cdot, t)\right\|_{H^{-1}(\Omega)}^{2} \\
& \quad \leq\left\|u_{0}-u_{h \tau}(\cdot, 0)\right\|_{H^{-1}(\Omega)}^{2}+\left\|\mathcal{R}\left(u_{h \tau}\right)\right\|_{X_{t}^{\prime}}^{2}+\left\|u-u_{h \tau}\right\|_{X_{t}^{\prime}}^{2}
\end{aligned}
$$


Proof. For a.e. $t \in(0, T)$, we denote by $W(\cdot, t) \in H_{0}^{1}(\Omega)$ the solution to

$$
(\nabla W(\cdot, t), \nabla \psi)=\left(\left(u-u_{h \tau}\right)(\cdot, t), \psi\right) \quad \forall \psi \in H_{0}^{1}(\Omega) .
$$

The existence and uniqueness of $W(\cdot, t)$ follow from the Riesz representation theorem. Moreover, since $u, u_{h \tau} \in Z$, there holds $W \in X$. Using (A.10), it is inferred that

$$
\begin{aligned}
\|\nabla W(\cdot, t)\|_{L^{2}(\Omega)} & =\sup _{\psi \in H_{0}^{1}(\Omega),\|\nabla \psi\|_{L^{2}(\Omega)}=1}(\nabla W(\cdot, t), \nabla \psi) \\
& =\sup _{\psi \in H_{0}^{1}(\Omega),\|\nabla \psi\|_{L^{2}(\Omega)}=1}\left(\left(u-u_{h \tau}\right)(\cdot, t), \psi\right) \\
& =\left\|\left(u-u_{h \tau}\right)(\cdot, t)\right\|_{H^{-1}(\Omega)} .
\end{aligned}
$$

This duality technique is rather standard; see 9 and the references therein. Its origins can be traced back at least to the elliptic projection of Wheeler [53. In some aspects, it is close to the elliptic reconstruction of Makridakis and Nochetto 31; however, in 31 it is used to restore optimal order of the a posteriori estimate in $L^{\infty}\left(0, T ; L^{2}(\Omega)\right)$, whereas here we employ it to obtain a bound on an energy-like norm.

Taking $\varphi=W 1_{(0, t)}$ with $1_{(0, t)}$ the characteristic function of the interval $(0, t)$ in definition (3.1) and using (A.11) and the Young inequality, it is inferred that

$$
\left\langle\mathcal{R}\left(u_{h \tau}\right), W\right\rangle_{X_{t}^{\prime}, X_{t}} \leq\left\|\mathcal{R}\left(u_{h \tau}\right)\right\|_{X_{t}^{\prime}}\left\|u-u_{h \tau}\right\|_{X_{t}^{\prime}} \leq \frac{1}{2}\left\|\mathcal{R}\left(u_{h \tau}\right)\right\|_{X_{t}^{\prime}}^{2}+\frac{1}{2}\left\|u-u_{h \tau}\right\|_{X_{t}^{\prime}}^{2} .
$$

Moreover,

$$
\begin{aligned}
\left\langle\mathcal{R}\left(u_{h \tau}\right), W\right\rangle_{X_{t}^{\prime}, X_{t}}= & \int_{0}^{t}\left\langle\partial_{t}\left(u-u_{h \tau}\right), W\right\rangle(s) \mathrm{d} s \\
& +\int_{0}^{t}\left(\nabla \beta(u)-\nabla \beta\left(u_{h \tau}\right), \nabla W\right)(s) \mathrm{d} s=: \mathfrak{R}_{1}+\mathfrak{R}_{2} .
\end{aligned}
$$

Recalling (A.10), and since $u-u_{h \tau} \in H^{1}\left(0, T ; H^{-1}(\Omega)\right)$, there holds $\partial_{t} W \in X$ and, for a.e. $s \in(0, T), \partial_{t} W(\cdot, s)$ satisfies in a weak sense

$$
\begin{aligned}
-\nabla \cdot\left(\nabla \partial_{t} W(\cdot, s)\right) & =\partial_{t}\left(u-u_{h \tau}\right)(\cdot, s) & & \text { in } \Omega, \\
\partial_{t} W(\cdot, s) & =0 & & \text { on } \partial \Omega .
\end{aligned}
$$

Thus, it follows from the definition A.10 of $W$ and from the norm characterization A.11 that

$$
\begin{aligned}
\mathfrak{R}_{1} & =\int_{0}^{t}\left(\partial_{t} \nabla W, \nabla W\right)(s) \mathrm{d} s=\frac{1}{2}\left(\|\nabla W(\cdot, t)\|_{L^{2}(\Omega)}^{2}-\|\nabla W(\cdot, 0)\|_{L^{2}(\Omega)}^{2}\right) \\
& =\frac{1}{2}\left(\left\|\left(u-u_{h \tau}\right)(\cdot, t)\right\|_{H^{-1}(\Omega)}^{2}-\left\|u_{0}-u_{h \tau}(\cdot, 0)\right\|_{H^{-1}(\Omega)}^{2}\right) .
\end{aligned}
$$

Invoking again the definition (A.10) and using the fact that $\beta$ is nondecreasing and $L_{\beta}$-Lipschitz continuous, there holds

$$
\begin{aligned}
\mathfrak{R}_{2} & =\int_{0}^{t}\left(u-u_{h \tau}, \beta(u)-\beta\left(u_{h \tau}\right)\right)(s) \mathrm{d} s \\
& \geq \frac{1}{L_{\beta}} \int_{0}^{t}\left(\beta(u)-\beta\left(u_{h \tau}\right), \beta(u)-\beta\left(u_{h \tau}\right)\right)(s) \mathrm{d} s \\
& =\frac{1}{L_{\beta}}\left\|\beta(u)-\beta\left(u_{h \tau}\right)\right\|_{Q_{t}}^{2} .
\end{aligned}
$$


The conclusion follows using the inequalities (A.12, A.14), and A.15) in equation $\mathrm{A.13}$.

Corollary A.4 (Application of the Gronwall lemma). Under the assumptions of Lemma A.3, there holds

$$
\begin{aligned}
\left\|u-u_{h \tau}\right\|_{X^{\prime}}^{2} & \leq\left(e^{T}-1\right)\left\|u_{0}-u_{h \tau}(\cdot, 0)\right\|_{H^{-1}(\Omega)}^{2} \\
& +\int_{0}^{T}\left(\left\|\mathcal{R}\left(u_{h \tau}\right)\right\|_{X_{t}^{\prime}}^{2}+\int_{0}^{t}\left\|\mathcal{R}\left(u_{h \tau}\right)\right\|_{X_{s}^{\prime}}^{2} e^{t-s} \mathrm{~d} s\right) \mathrm{d} t \\
& -\frac{2}{L_{\beta}} \int_{0}^{T}\left(\left\|\beta(u)-\beta\left(u_{h \tau}\right)\right\|_{Q_{t}}^{2}+\int_{0}^{t}\left\|\beta(u)-\beta\left(u_{h \tau}\right)\right\|_{Q_{s}}^{2} e^{t-s} \mathrm{~d} s\right) \mathrm{d} t .
\end{aligned}
$$

Proof. Using (A.9) followed by the Gronwall lemma

$$
\xi(t) \leq \alpha(t)+\int_{0}^{t} \xi(s) \mathrm{d} s \Longrightarrow \xi(t) \leq \alpha(t)+\int_{0}^{t} \alpha(s) e^{t-s} \mathrm{~d} s
$$

with $\xi(t):=\left\|\left(u-u_{h \tau}\right)(\cdot, t)\right\|_{H^{-1}(\Omega)}^{2}$ and $\alpha(t):=\left\|u_{0}-u_{h \tau}(\cdot, 0)\right\|_{H^{-1}(\Omega)}^{2}+\left\|\mathcal{R}\left(u_{h \tau}\right)\right\|_{X_{t}^{\prime}}^{2}-$ $\frac{2}{L_{\beta}}\left\|\beta(u)-\beta\left(u_{h \tau}\right)\right\|_{Q_{t}}^{2}$, it is inferred, for a.e. $t \in(0, T)$,

$$
\begin{aligned}
& \left\|\left(u-u_{h \tau}\right)(\cdot, t)\right\|_{H^{-1}(\Omega)}^{2} \leq e^{t}\left\|u_{0}-u_{h \tau}(\cdot, 0)\right\|_{H^{-1}(\Omega)}^{2}+\left\|\mathcal{R}\left(u_{h \tau}\right)\right\|_{X_{t}^{\prime}}^{2} \\
& +\int_{0}^{t}\left\|\mathcal{R}\left(u_{h \tau}\right)\right\|_{X_{s}^{\prime}}^{2} e^{t-s} \mathrm{~d} s-\frac{2}{L_{\beta}}\left(\left\|\beta(u)-\beta\left(u_{h \tau}\right)\right\|_{Q_{t}}^{2}+\int_{0}^{t}\left\|\beta(u)-\beta\left(u_{h \tau}\right)\right\|_{Q_{s}}^{2} e^{t-s} \mathrm{~d} s\right) .
\end{aligned}
$$

The assertion follows by integrating over the interval $(0, T)$.

We are now ready to prove Theorem 5.2

Proof of Theorem 5.2. Using (A.9) with $t=T$ and adding $\left\|u-u_{h \tau}\right\|_{X^{\prime}}^{2}$ to both sides we infer

$$
\begin{aligned}
\mathfrak{L} & :=\frac{2}{L_{\beta}}\left\|\beta(u)-\beta\left(u_{h \tau}\right)\right\|_{Q_{T}}^{2}+\left\|u-u_{h \tau}\right\|_{X^{\prime}}^{2}+\left\|\left(u-u_{h \tau}\right)(\cdot, T)\right\|_{H^{-1}(\Omega)}^{2} \\
& \leq\left\|u_{0}-u_{h \tau}(\cdot, 0)\right\|_{H^{-1}(\Omega)}^{2}+\left\|\mathcal{R}\left(u_{h \tau}\right)\right\|_{X^{\prime}}^{2}+2\left\|u-u_{h \tau}\right\|_{X^{\prime}}^{2} .
\end{aligned}
$$

Using Corollary A.4 to estimate the last term in the right-hand side we obtain

$$
\begin{aligned}
\mathfrak{L} \leq & \left(2 e^{T}-1\right)\left\|u_{0}-u_{h \tau}(\cdot, 0)\right\|_{H^{-1}(\Omega)}^{2}+\left\|\mathcal{R}\left(u_{h \tau}\right)\right\|_{X^{\prime}}^{2} \\
& +2 \int_{0}^{T}\left(\left\|\mathcal{R}\left(u_{h \tau}\right)\right\|_{X_{t}^{\prime}}^{2}+\int_{0}^{t}\left\|\mathcal{R}\left(u_{h \tau}\right)\right\|_{X_{s}^{\prime}}^{2} e^{t-s} \mathrm{~d} s\right) \mathrm{d} t \\
& -\frac{4}{L_{\beta}} \int_{0}^{T}\left(\left\|\beta(u)-\beta\left(u_{h \tau}\right)\right\|_{Q_{t}}^{2}+\int_{0}^{t}\left\|\beta(u)-\beta\left(u_{h \tau}\right)\right\|_{Q_{s}}^{2} e^{t-s} \mathrm{~d} s\right) \mathrm{d} t .
\end{aligned}
$$

The conclusion follows multiplying both sides by $L_{\beta} / 2$ and rearranging the terms.

\section{REFERENCES}

[1] H. W. Alt and S. Luckhaus, Quasilinear elliptic-parabolic differential equations, Math. Z. 183 (1983), no. 3, 311-341, DOI 10.1007/BF01176474. MR706391 (85c:35059)

[2] G. Amiez and P.-A. Gremaud, On a numerical approach to Stefan-like problems, Numer. Math. 59 (1991), no. 1, 71-89, DOI 10.1007/BF01385771. MR1103754 (92c:65102)

[3] B. Andreianov, M. Bendahmane, and K. H. Karlsen, Discrete duality finite volume schemes for doubly nonlinear degenerate hyperbolic-parabolic equations, J. Hyperbolic Differ. Equ. 7 (2010), no. 1, 1-67, DOI 10.1142/S0219891610002062. MR2646796 (2011d:65241) 
[4] L. A. Baughman and N. J. Walkington, Co-volume methods for degenerate parabolic problems, Numer. Math. 64 (1993), no. 1, 45-67, DOI 10.1007/BF01388680. MR1191322 (93j:65147)

[5] M. Bebendorf, A note on the Poincaré inequality for convex domains, Z. Anal. Anwendungen 22 (2003), no. 4, 751-756, DOI 10.4171/ZAA/1170. MR2036927(2004k:26025)

[6] G. Beckett, J. A. Mackenzie, and M. L. Robertson, A moving mesh finite element method for the solution of two-dimensional Stefan problems, J. Comput. Phys. 168 (2001), no. 2, 500-518, DOI 10.1006/jcph.2001.6721. MR.1826524 (2002a:80004)

[7] P. Benilan and P. Wittbold, On mild and weak solutions of elliptic-parabolic problems, Adv. Differential Equations 1 (1996), no. 6, 1053-1073. MR.1409899 (97e:35119)

[8] D. Braess and J. Schöberl, Equilibrated residual error estimator for edge elements, Math. Comp. 77 (2008), no. 262, 651-672, DOI 10.1090/S0025-5718-07-02080-7. MR2373174 $(2008 \mathrm{~m}: 65313)$

[9] C. Cancès, L. S. Pop, and M. Vohralík, An a posteriori error estimate for vertex-centered finite volume discretizations of immiscible incompressible two-phase flow, Math. Comp. 83 (2014), 153-188, DOI 10.1090/S0025-5718-2013-02723-8. MR3120585

[10] L. Cermák and M. Zlámal, Transformation of dependent variables and the finite element solution of nonlinear evolution equations, Internat. J. Numer. Methods Engrg. 15 (1980), no. 1, 31-40, DOI 10.1002/nme.1620150104. MR554438 (80i:65112)

[11] P. G. Ciarlet, The Finite Element Method for Elliptic Problems, North-Holland Publishing Co., Amsterdam, 1978. Studies in Mathematics and its Applications, Vol. 4. MR0520174 $(58$ \#25001)

[12] J. F. Ciavaldini, Analyse numérique d'un problème de Stefan à deux phases par une méthode d'éléments finis (French, with English summary), SIAM J. Numer. Anal. 12 (1975), 464-487. MR0391741 (52 \#12561)

[13] P. Destuynder and B. Métivet. Explicit error bounds in a conforming finite element method. Math. Comp., 68(228):1379-1396, 1999.

[14] V. Dolejší, A. Ern, and M. Vohralík, A framework for robust a posteriori error control in unsteady nonlinear advection-diffusion problems, SIAM J. Numer. Anal. 51 (2013), no. 2, 773-793, DOI 10.1137/110859282. MR3033032

[15] L. El Alaoui, A. Ern, and M. Vohralík, Guaranteed and robust a posteriori error estimates and balancing discretization and linearization errors for monotone nonlinear problems, Comput. Methods Appl. Mech. Engrg. 200 (2011), no. 37-40, 2782-2795, DOI 10.1016/j.cma.2010.03.024. MR2811915(2012e:65267)

[16] C. M. Elliott, On the finite element approximation of an elliptic variational inequality arising from an implicit time discretization of the Stefan problem, IMA J. Numer. Anal. 1 (1981), no. 1, 115-125, DOI 10.1093/imanum/1.1.115. MR607251(82d:65072)

[17] A. Ern and M. Vohralík, Flux reconstruction and a posteriori error estimation for discontinuous Galerkin methods on general nonmatching grids (English, with English and French summaries), C. R. Math. Acad. Sci. Paris 347 (2009), no. 7-8, 441-444, DOI 10.1016/j.crma.2009.01.017. MR2537245

[18] A. Ern and M. Vohralík, A posteriori error estimation based on potential and flux reconstruction for the heat equation, SIAM J. Numer. Anal. 48 (2010), no. 1, 198-223, DOI 10.1137/090759008. MR2608366 (2011d:65281)

[19] A. Ern and M. Vohralík, Adaptive inexact Newton methods with a posteriori stopping criteria for nonlinear diffusion PDEs, SIAM J. Sci. Comput. 35 (2013), no. 4, A1761-A1791, DOI 10.1137/120896918. MR 3072765

[20] R. Eymard, T. Gallouët, and R. Herbin, Finite volume methods, Handbook of numerical analysis, Vol. VII, North-Holland, Amsterdam, 2000, pp. 713-1020. MR.1804748 (2002e:65138)

[21] R. Eymard, T. Gallouët, D. Hilhorst, and Y. Naït Slimane, Finite volumes and nonlinear diffusion equations (English, with English and French summaries), RAIRO Modél. Math. Anal. Numér. 32 (1998), no. 6, 747-761. MR1652593 (99j:65166)

[22] A. Friedman, The Stefan problem in several space variables, Trans. Amer. Math. Soc. 133 (1968), 51-87. MR0227625 (37 \#3209)

[23] D. Hilhorst and M. Vohralík, A posteriori error estimates for combined finite volumefinite element discretizations of reactive transport equations on nonmatching grids, Comput. Methods Appl. Mech. Engrg. 200 (2011), no. 5-8, 597-613, DOI 10.1016/j.cma.2010.08.017. MR2749021(2012d:65207) 
[24] W. Jäger and J. Kačur, Solution of porous medium type systems by linear approximation schemes, Numer. Math. 60 (1991), no. 3, 407-427, DOI 10.1007/BF01385729. MR.1137200 (92j:76092)

[25] J. W. Jerome and M. E. Rose, Error estimates for the multidimensional two-phase Stefan problem, Math. Comp. 39 (1982), no. 160, 377-414, DOI 10.2307/2007320. MR669635 (84h:65097)

[26] P. Jiránek, Z. Strakoš, and M. Vohralík, A posteriori error estimates including algebraic error and stopping criteria for iterative solvers, SIAM J. Sci. Comput. 32 (2010), no. 3, 1567-1590, DOI 10.1137/08073706X. MR2652091(2011e:65233)

[27] S. L. Kamenomostskaja, On Stefan's problem (Russian), Mat. Sb. (N.S.) 53 (95) (1961), 489-514. MR0141895(25 \#5292)

[28] C. T. Kelley and J. Rulla, Solution of the time discretized Stefan problem by Newton's method, Nonlinear Anal. 14 (1990), no. 10, 851-872, DOI 10.1016/0362-546X(90)90025-C. MR:1055534(92b:65084)

[29] P. Ladevèze. Comparaison de modèles de milieux continus. Ph.D. thesis, Université Pierre et Marie Curie (Paris 6), 1975.

[30] R. Luce and B. I. Wohlmuth, A local a posteriori error estimator based on equilibrated fluxes, SIAM J. Numer. Anal. 42 (2004), no. 4, 1394-1414 (electronic), DOI 10.1137/S0036142903433790. MR2114283 (2006d:65122)

[31] C. Makridakis and R. H. Nochetto, Elliptic reconstruction and a posteriori error estimates for parabolic problems, SIAM J. Numer. Anal. 41 (2003), no. 4, 1585-1594, DOI 10.1137/S0036142902406314. MR2034895 (2004k:65157)

[32] G. H. Meyer, Multidimensional Stefan problems, SIAM J. Numer. Anal. 10 (1973), 522-538. MR0331807 (48 \#10139)

[33] R. H. Nochetto, Error estimates for multidimensional singular parabolic problems, Japan J. Appl. Math. 4 (1987), no. 1, 111-138, DOI 10.1007/BF03167758. MR899207 (89c:65107)

[34] R. H. Nochetto, M. Paolini, and C. Verdi, An adaptive finite element method for two-phase Stefan problems in two space dimensions. I. Stability and error estimates, Math. Comp. $\mathbf{5 7}$ (1991), no. 195, 73-108, S1-S11, DOI 10.2307/2938664. MR.1079028(92a:65322)

[35] R. H. Nochetto, M. Paolini, and C. Verdi, An adaptive finite element method for twophase Stefan problems in two space dimensions. II. Implementation and numerical experiments, SIAM J. Sci. Statist. Comput. 12 (1991), no. 5, 1207-1244, DOI 10.1137/0912065. MR.1114983(92f:65138)

[36] R. H. Nochetto, A. Schmidt, and C. Verdi, A posteriori error estimation and adaptivity for degenerate parabolic problems, Math. Comp. 69 (2000), no. 229, 1-24, DOI 10.1090/S00255718-99-01097-2. MR.1648399 (2000i:65136)

[37] R. H. Nochetto and C. Verdi, The combined use of a nonlinear Chernoff formula with a regularization procedure for two-phase Stefan problems, Numer. Funct. Anal. Optim. 9 (1987/88), no. 11-12, 1177-1192, DOI 10.1080/01630568808816279. MR936337(89b:65205)

[38] F. Otto, L $L^{1}$-contraction and uniqueness for quasilinear elliptic-parabolic equations, J. Differential Equations 131 (1996), no. 1, 20-38, DOI 10.1006/jdeq.1996.0155. MR1415045 (97i:35125)

[39] L. E. Payne and H. F. Weinberger, An optimal Poincaré inequality for convex domains, Arch. Rational Mech. Anal. 5 (1960), 286-292 (1960). MR0117419 (22 \#8198)

[40] M. Picasso, An adaptive finite element algorithm for a two-dimensional stationary Stefanlike problem, Comput. Methods Appl. Mech. Engrg. 124 (1995), no. 3, 213-230, DOI 10.1016/0045-7825(95)00793-Z. MR.1343078 (96f:65157)

[41] M. Picasso, Adaptive finite elements for a linear parabolic problem, Comput. Methods Appl. Mech. Engrg. 167 (1998), no. 3-4, 223-237, DOI 10.1016/S0045-7825(98)00121-2. MR.1673951 (2000b:65188)

[42] I. S. Pop, M. Sepúlveda, F. A. Radu, and O. P. Vera Villagrán. Error estimates for the finite volume discretization for the porous medium equation. J. Comput. Appl. Math., 234(7):21352142, 2010.

[43] W. Prager and J. L. Synge, Approximations in elasticity based on the concept of function space, Quart. Appl. Math. 5 (1947), 241-269. MR0025902 (10,81b)

[44] A. Quarteroni and A. Valli, Numerical Approximation of Partial Differential Equations, Springer Series in Computational Mathematics, vol. 23, Springer-Verlag, Berlin, 1994. MR $1299729(95 \mathrm{i}: 65005)$ 
[45] S. Repin, A Posteriori Estimates for Partial Differential Equations, Radon Series on Computational and Applied Mathematics, vol. 4, Walter de Gruyter GmbH \& Co. KG, Berlin, 2008. MR2458008 (2010b:35004)

[46] R. Verfürth, A posteriori error estimates for nonlinear problems. $L^{r}\left(0, T ; L^{\rho}(\Omega)\right)$-error estimates for finite element discretizations of parabolic equations, Math. Comp. 67 (1998), no. 224, 1335-1360, DOI 10.1090/S0025-5718-98-01011-4. MR1604371 (99b:65120)

[47] R. Verfürth, A posteriori error estimates for nonlinear problems: $L^{r}\left(0, T ; W^{1, \rho}(\Omega)\right)$ error estimates for finite element discretizations of parabolic equations, Numer. Methods Partial Differential Equations 14 (1998), no. 4, 487-518, DOI 10.1002/(SICI)10982426(199807)14:4〈487::AID-NUM4〉3.0.CO;2-G. MR 1627578 (99g:65099)

[48] R. Verfürth, A posteriori error estimates for finite element discretizations of the heat equation, Calcolo 40 (2003), no. 3, 195-212, DOI 10.1007/s10092-003-0073-2. MR2025602 (2005f:65131)

[49] R. Verfürth, Robust a posteriori error estimates for stationary convection-diffusion equations, SIAM J. Numer. Anal. 43 (2005), no. 4, 1766-1782 (electronic), DOI 10.1137/040604261. MR2182149(2007d:65116)

[50] M. Vohralík, A posteriori error estimation in the conforming finite element method based on its local conservativity and using local minimization (English, with English and French summaries), C. R. Math. Acad. Sci. Paris 346 (2008), no. 11-12, 687-690, DOI 10.1016/j.crma.2008.03.006. MR2423279(2009c:65326)

[51] M. Vohralík, Guaranteed and fully robust a posteriori error estimates for conforming discretizations of diffusion problems with discontinuous coefficients, J. Sci. Comput. 46 (2011), no. 3, 397-438, DOI 10.1007/s10915-010-9410-1. MR2765501 (2012a:65297)

[52] J. A. Wheeler, Permafrost design for the trans-Alaska, in P. T. Boggs, editor, Moving Boundary Problems, Academic Press, New York, 1978.

[53] M. F. Wheeler, A priori $L_{2}$ error estimates for Galerkin approximations to parabolic partial differential equations, SIAM J. Numer. Anal. 10 (1973), 723-759. MR0351124 (50 \#3613)

Université Montpellier 2, I3M, 34057 Montpellier, France

E-mail address: daniele.di-pietro@univ-montp2.fr

UPMC Université PAris 06, UMR 7598, Laboratoire JaCQUes-Louis Lions, 75005, PARIS, France - AND - CNRS, UMR 7598, Laboratoire JaCques-Louis Lions, 75005, PARis, France Current address: INRIA Paris-Rocquencourt, B.P. 105, 78153 Le Chesnay, France

E-mail address: martin.vohralik@inria.fr

UPMC Université, Paris 06, UMR 7598, Laboratoire JaCques-Louis Lions, 75005, Paris, France - And - IFP Energies nouvelles, Department of Applied Mathematics, 1 \& 4 avenue Bois Préau, 92852 Rueil-Malmaison, France

E-mail address: yousef@ann.jussieu.fr 